PALEO

Revue d'archéologie préhistorique

19 | 2007

Spécial table ronde ( 1 ère partie) : Le Gravettien : entités régionales d'une paléoculture européenne, Les Eyzies, juillet 2004

\title{
L'exploitation du Lièvre variable à La Madeleine (Dordogne, France) et le statut d'un petit gibier au Dryas ancien
}

Arctic Hare Exploitation in La Madeleine and the Economic Status of a Small

Game during the Ancient Dryas

Laure Fontana et François-Xavier Chauvière

\section{(2) OpenEdition}

Journals

Édition électronique

URL : https://journals.openedition.org/paleo/661

DOI : 10.4000/paleo.661

ISSN : 2101-0420

Éditeur

SAMRA

Édition imprimée

Date de publication : 30 décembre 2007

Pagination : 303-336

ISSN : $1145-3370$

Référence électronique

Laure Fontana et François-Xavier Chauvière, "L'exploitation du Lièvre variable à La Madeleine (Dordogne, France) et le statut d'un petit gibier au Dryas ancien », PALEO [En ligne], 19 | 2007, mis en ligne le 23 avril 2009, consulté le 26 juin 2021. URL : http://journals.openedition.org/paleo/661 ; DOI https://doi.org/10.4000/paleo.661

\section{(c) (i) (9)}

PALEO est mis à disposition selon les termes de la licence Creative Commons Attribution - Pas d'Utilisation Commerciale - Pas de Modification 4.0 International. 


\title{
L'EXPLOITATION DU LIÈVRE VARIABLE A LA MADELEINE (Dordogne, France) ET LE STATUT D'UN PETIT GIBIER AU DRYAS ANCIEN
}

\author{
Laure FONTANA ${ }^{(1)}$ et François-Xavier CHAUVIÈRE ${ }^{(2)}$
}

\begin{abstract}
Résumé : Cette étude présente les résultats de l'analyse des restes osseux et dentaires de Lepus timidus issus des couches du Magdalénien IV de La Madeleine (fouilles Peyrony et Bouvier). II s'agissait de caractériser l'exploitation du Lièvre variable et c'est pourquoi l'ensemble des restes, transformés ou non, a été pris en compte au sein d'une étude intégrée. La représentativité des deux collections, ancienne et récente, est variable, ce qui ne limite pas nécessairement le potentiel informatif des deux séries. La part du Lièvre au sein des espèces chassées, difficile à apprécier, se situe probablement entre 7 et $20 \%$ (en NR). Si l'étude de l'exploitation des carcasses met en évidence le prélèvement de plusieurs produits, alimentaires ou non, leur part respective et leur consommation sont plus difficiles à préciser. L'hypothèse d'une éventuelle recherche prioritaire de la fourrure est discutée.
\end{abstract}

Mots-clés : Lièvre variable, modalités d'exploitation, Magdalénien IV, sud-ouest de la France.

Key-words: Arctic Hare, Exploitation patterns, Magdalenian IV, Southwestern France.

\section{Abridged english version \\ ARCTIC HARE EXPLOITATION IN LA MADELEINE AND THE ECONOMIC STATUS OF A SMALL GAME DURING THE ANCIENT DRYAS.}

Once we made a first synthesis about Arctic Hare (Lepus timidus) exploitation, from data of twenty-three Magdalenian sites of western Europe (Fontana 2004), it was essential that we study Arctic Hare remains from La Madeleine, for geographical and chronological reasons. So we made the analysis of Arctic Hare remains from the Magdalenian IV levels of La Madeleine, to characterize this small game exploitation and to contribute to identifying the Arctic Hare economical status.

La Madeleine (Tursac, Dordogne), discovered in 1863 by E. Lartet and H. Christy, is a large rock shelter, 250 meters long, located at the foot of a 45 meters overhanging cliff, facing South and extending over the right side of the Vézère river (fig. 1). After E. Lartet, L. Girod, E. Massenat (1895) and E. Rivière (1901), D. Peyrony made very large excavations, from 1910 to 1913 (fig. 2), and when he started again in 1926 in the eastern part, he discovered the child burial (Bouvier 1972). There are three recognized geologic strata, identified from six stratigraphic profiles and related to three archaeological units (fig. 3a). The lower unit, related to the Magdalenian IV (Capitan \& Peyrony 1928, p. 17), the middle unit to the Magdalenian V, the upper unit to the Magdalenian VI. Between 1967 and 1983 , J.-M. Bouvier made a drilling and a 50 square meters excavation in the western part (cf. fig. 2) that was still undisturbed. Inside the eighteen archaeological levels he identified, Bouvier isolated five Magdalenian IV levels (30 to 26), six Magdalenian V (25 to 20) and seven Magdalenian VI (19 to 13) levels (fig. 3b). Four radiocarbon dates were obtained from bones collected in four levels (19, 21 and 25,26 ) and as the level $26-13440+/-300$ BP (Ly 919) - was the only one and the most recent belonging to the Magdalenian IV (Bouvier 1973, 1977, 1979), we still do not have any date for the former occupation in La Madeleine.

Hare remains from Peyrony and Bouvier series come from different parts (East and West, large and small) of the site. They have been collected, presened and studied in a different way. It is clear that Peyrony has collected only complete bones and not the smallest ones. More over, we discovered that a part of the series had been lost after the first anatomical part sorting: vertebrae, ribs, stemum, maxillars, all the tarsals (except the calcaneus), all the carpals, the sesamoids, patellae, the second and the third phalanges, the first anterior phalanges. These anatomical parts are present in the Bouvier series and we also discovered some of them, still in a little piece of soil presened from Peyrony excavations. Concerning Bouvier's excavations, if almost every remain has been collected (sieving), it is not possible to know if the fact that the remains come from an outlying part of the site makes this assemblage less representative. The remains are actually preserved in the National Museum of Prehistory of Les Eyzies (France).

(1) CNRS, UMR 6636 (LAMPEA). Maison Méditerranéenne des Sciences de l'Homme, 5 rue du Château de l'Horloge BP 647, 13094 Aix-en-Provence cedex - Ifontana@mmsh.univ-aix.fr

(2) Institut de Préhistoire et des sciences de l'Antiquité. Université de Neuchâtel. Office et Musée d'Archéologie. LATENIUM, Espace Paul Vouga CH - 2068 Hauterive - Université Jean Moulin Lyon III - francois-xavier.chauviere@unine.ch 
The great majority of Hare remains (1035) belongs to the Magdalenian IV levels (tabl. 1, 2). 99\% of identified remains belong to the Reindeer, the Horse and the Arctic Hare whose place is different according to the series (tabl. 3, 4, 5 ; fig. 4): 5, 27\% in the Bouvier series (283 remains representing at least 10 hares) and $37 \%$ in the Peyrony assemblage (752 remains representing at least 41 hares). It seems that this difference comes from a better attention paid to Hare remains comparing to Reindeer and Horse remains during Peyrony excavations: Hare was probably less represented. So we can just estimate the Hare place in La Madeleine between 6 and 25\%.

There is no clear evidence for natural accumulation of Hare bones in La Madeleine, but, on the contrary, many pieces of evidence of Hare exploitation during the Magdalenian IV. 46 bone remains bear cutmarks that are related to carcass processing activities, including the manufacture of blanks and objects (tabl. 6). More than $95 \%$ of individuals are adults and the skeletal part frequencies are also similar to the ones documented in human accumulations (Hockett 1991, 1992, 1994 ; Morel \& Müller 1997 ; Speth 2000 ; Hockett \& Haws 2002 ; Fontana 2003 ; Cochard 2004): presence of all the skeletal parts, similar proportions of forelimb and hind limb bones, abundance of limb bones, scapulae and pelvis, high frequency of metapodials (specially metatarsals), very low frequency of vertebrae, ribs, stemum and skull, high frequency of the mandible comparing to the skull (tabl. 7). Finally, the scarcity of complete long bones (only metapodials) as the high rate of breakage in the Bouvier series is another witness of a human accumulation.

What did people remove and consume from hares carcasses, how, and with any priority?

Hare skeletal part frequency of the two series is overall similar and looks like many others from other Magdalenian sites (tabl. 8, 9, 10, fig. $5,6,7)$. Its homogeneity is not as remarkable as in Gazel Cave but it is still important comparing to large herbivorous skeletal part frequency. So, entire carcasses of adult hares were carried to the cave for processing and probably consumption, as we will demonstrate now.

46 Hare bones bear cutmarks and other ones like scraping, severing and grooving (tabl. 11, cf. tabl. 6 and fig. 8). The cutmarks identified on 25 remains are related to dismembering (fig. 9), butchering (fig. 10) and skinning. If disarticulation marks are common, butchering marks are a little bit different from what we expected (cf. fig. 8): there is no mark on the neck of the scapula, while marks frequency is very high on the four sites documented, and there are a lot of marks on the radius, what is very unusual. The skinning marks are very rare and noticed on a mandible (cf. fig. 8) and on a metatarsal IV whose diaphyse is covered by numerous transversal and sharp marks (fig. 11). There are, on 16 long bones (fig.12, 13), specially radii (5) and tibiae (4) (cf. tabl. 6), some marks of scraping on the whole diaphyse (cf. fig. 8). If that this process is related to the removal of the meat, it was probably not the final aim, since it concerned bones without much meat. But if the final aim was to clean the surface, we wonder why these bones have not been used. $4 \%$ of Hare remains bear marks, that is not many, if we compare to Romanelli Cave (6,3\%, Tagliacozzo \& Fiore 1998), Bois Ragot (nearly $8 \%$, Cochard 2004), Champréveyres (nearly 13\%, Morel \& Müller 1998) and Robin Hood Cave (22\%, Charles \& Jacobi 1994). But it's a lot if we compare to Gazel Cave (0,5\%, Fontana 2003) and other sites where no remain does not bear any mark, like Belvis (Fontana 1998), Les Eglises (Delpech \& Le Gall 1983) or Combe-Saunière (Castel 1999). The marks confirm the fur removal, first suggested by the absence of caudal vertebrae and patellae, but we still ignore if it concerned every carcass or just some of them. Then, as we found no buming mark, it is not possible to demonstrate roasting practice and to be sure that meat was cooked.

Six bones bear marks of grooving and sawing using a sharp tool, what is related to the manufacture of little tools and ornaments (tabl. 12). Indeed, four long bones bear, on their anterior surface, traces of grooving which were made to obtain rectilinear sticks (fig. 14). Manufacturing needles was the aim of such a processing which probably took part in situ (fig. 15). A metatarsal III from the Bouvier series (level 28) was split up into two parts, also with a sharp tool (fig. 16). The small cylinder obtained could have been modified into a tube or being used for the manufacturing of an imitation of a test of dentalium, like in Petersfels, in Germany (Albrecht \& al. 1983). Finally, a tibia shaft (with proximal end, cf. tabl. 6) from the Peyrony series has a specific oval hole located on the crest of the tibia (fig. 17), which could be due to a chemical dissolving of the bone. Even if the manufacture of needles was the main goal of Hare bones exploitation, it was not an important production, as in the other Magdalenian sites where it is documented (tabl. 13): at the scale of the site, the manufacture of Hare needles can even be considered as a detail, since needles were first produced from Reindeer and Horse bones (Capitan \& Peyrony 1928).

How have bones been fractured and what for? Inside the two series, long bones are almost only shaft fragments (with or without their distal end) and the metapodials are the only ones not to be broken (cf. tabl. 7). In the Peyrony series, the broken long bones are all complete cylinders whose length is varied (fig. 18.1, 18.2), that differs from those of the Bouvier series which are more diverse (epiphyses, shaft fragments, with or without any end) (fig. 18.3 to 18.6) and smaller. So, long limb bones were systematically highly broken (metapodials excepted), and the great majority of them still bear characteristics of green bone fractures (Morlan 1984; Johnson 1985) (fig. 18.2, 18.3, 18.5), some radii also bearing marks of dry fractures (fig. 18.1 \& 18.6). If fracture features, already described, seem to be more related to "fracture par flexion" (cf. fig. 18), some shaft fragments document also the use of percussion. But no evidence of human tooth fracture has been noticed, like documented for the rabbits in Spanish sites (Perez Ripoll 1993, 2004, 2005/2006).

Studying breakage patterns is essential to deal with the subject of marrow and grease extraction. We notice that in La Madeleine, Hare shaft cylinders or epiphyses, which would attest to the marrow removal (Hockett 1991, 1995; Morel \& Müller 1997; Perez Ripoll 1993; Schmitt 1990), are rare. Indeed, these are limb bones diaphyses with one of the two ends, which are the most numerous. It documents another kind of limb bone breakage, that is also documented in other sites (Hockett \& Bicho 2000; Perez Ripoll 2002). Additionally, the diaphyses have very different sizes. Consequently, if marrow has been extracted as some long bones breakage shows, it is not so obvious for all the large diaphyse fragments with one end (except the radii, if we consider their breakage in part as post-depositionnal). Does this breakage pattern reveal another way of marrow and grease removal (like a stock) or another aim?

Many products have been removed from the Hare carcasses: fur, meat, bones and marrow. If we can imagine that fur and marrow have been used or consumed, and demonstrate bone use, it is more difficult to know if raw meat has been consumed and if meat has been cooked. The data related to the marks and the breakage pattern indicates that every product has been removed for a small part: only two skinning marks, rare butchering marks testifying meat removal and six bones used for technical purposes. The only marrow removal could have been more systematic. Now if we consider the whole mammal resources, there is no doubt that Reindeer was the one which provided the great part of meat, marrow and bone. Additionally, we did not produce enough evidence that the priority was hare meat removal for consumption, on a site where a lot of reindeers and horses have been dismembered. Finally, it is also obvious that the fur of Arctic Hare had no equivalent. So, we may wonder if Hare exploitation was not focused on (white?) fur removal, as in Gazel Cave, considering the whole data, specially the choice of adult individuals. 
What did we learn about Arctic Hare exploitation during the old Dryas? If La Madeleine is one of the three sites, with Gazel Cave and Kesslerloch, documenting an important Arctic Hare hunting around $14500 \mathrm{BP}$, the part of this little game is smaller (1035 remains). Nevertheless, it seems that its place (probably third game after Reindeer and Horse), testifies that it was not just a complementary game whose procurement would have been more or less opportunistic. Indeed, if hares were, in La Madeleine, caught first or only to get some fur, this made its capture essential at the scale of the annual cycle. It is even possible that this peculiar fur was the favourite exploited skin, at least for some kind of clothes or bags, as we do not have many evidence of other fur procurement (very rare for the French Magdalenian of Southwestern France). This way of considering first the Arctic Hare as an animal exploited for its fur, and maybe the only one exploited for the fur, in the Magdalenian economic system, needs to be confirmed by studies of new or old sites, like Kesslerloch (Napierala, on going).

\section{INTRODUCTION}

Comprendre le statut économique des différents animaux chassés par les Magdaléniens d'Europe occidentale demeure l'un des objectifs de notre recherche, focalisée sur trois espèces qui semblent avoir eu un rôle-clé dans la société magdalénienne : le Renne, le Cheval et le Lièvre variable. Nous tentons de préciser la place de ces gibiers dans l'économie des groupes humains et d'identifier les modalités de leur exploitation en multipliant les études de collections anciennes et récentes (Fontana 2000 ; Fontana 2003 ; Fontana 2004 ; Chauvière et Fontana 2005 ; Chauvière et al. 2006 ; Chauvière et Fontana sous presse ; Fontana et Chauvière 2007 ; voir aussi Castel et al. 1998). Aujourd'hui, l'analyse des données semble souligner une exploitation particulière de chacun de ces trois gibiers et les questions ne se posent pas dans les mêmes termes selon qu'il s'agit du Renne, du Cheval ou du Lièvre variable. Si ce demier est l'un des rares animaux dont l'analyse des restes documente le prélèvement de la fourrure au Magdalénien, son exploitation apparaît comme hétérogène à tous points de vue (acquisition, exploitation et consommation). Tout l'intérêt de l'étude du Lièvre variable des niveaux du Magdalénien IV de La Madeleine (Tursac, Dordogne) réside précisément dans la possibilité de caractériser l'exploitation globale de cette ressource, par l'étude conjointe des déchets alimentaires et techniques et de la situer au sein des autres ressources animales. Le fait qu'il soit le seul gisement du sud-ouest français, hors Charente, à témoigner de l'exploitation de cet animal, renforce cet intérêt.

\section{1 - CADRE DE L'ÉTUDE}

\section{1 - Problématiques}

Au terme de notre première synthèse relative à l'exploitation du Lièvre variable (Lepus timidus), réalisée à partir des données de 23 sites magdaléniens d'Europe occidentale, plusieurs faits nous semblaient acquis (Fontana 2004). D'une part, le caractère exceptionnel et l'isolement géographique des sites où le Lièvre fut particulièrement recherché ainsi que la rareté, et peut-être la spécificité, d'une telle exploitation entre 15000 et 14000 BP. D'autre part, le fait que le statut de ce gibier ne fut pas celui d'un complément alimentaire et le fait que sa fourrure fut préférée, au moins en certains lieux et à certains moments, à celle des camivores et notamment du Renard commun et de I'Isatis. Nous avions noté une grande diversité des situations, qu'il s'agisse des modalités d'acquisition, d'exploitation ou encore de consommation, ainsi que l'originalité des données de la grotte Gazel (Fontana 2003).

Au regard de ce premier bilan, l'intérêt de l'étude des restes de Lièvre variable de La Madeleine était évident puisque ce gisement est l'un des trois sites d'Europe occidentale (avec la grotte Gazel dans l'Aude et Kesslerloch en Suisse) du Dryas ancien où le Lièvre variable fut l'un des principaux gibiers. Nous avons donc étudié les collections du Magdalénien IV de La Madeleine avec trois objectifs :

- caractériser l'exploitation du Lièvre en précisant sa part au sein des autres ressources animales, la nature de sa contribution économique, le mode de son acquisition ; - évaluer la contribution de cette étude à l'identification du statut économique du Lièvre variable en mettant en perspective les nouvelles données avec le bilan précédemment effectué ;

- comprendre comment l'exploitation du Lièvre à La Madeleine se situe par rapport à celle des lièvres des deux autres sites occupés au Dryas ancien.

\section{2 - Les fouilles et les études des restes fauniques}

Le site de La Madeleine a fait l'objet de multiples fouilles depuis sa découverte en 1863 par É. Lartet et H. Christy. Vaste abri sous-roche de 250 mètres de long au pied d'une falaise surplombante de 45 mètres, ce gisement orienté au sud s'étendait sur la rive droite de la Vézère (fig. 1). Si les fouilles d'É. Lartet dans la partie centrale puis celles de L. Girod et É. Massenat (vers 1895) et d'É. Rivière (vers 1901) plus à l'ouest (fig. 2) occupèrent au total une vaste surface, elles ne concemèrent pas l'intégralité du remplissage. Elles mirent au jour de nombreux restes fauniques, identifiés par É. Lartet (Lartet et Christy 1865-1875) et aujourd'hui dispersés. A partir de 1910, D. Peyrony commença ses travaux en évacuant les déblais des fouilles de ses prédécesseurs, ce qui lui permit d'exhumer une grande quantité de matériel et d'identifier les secteurs vierges. II commença ensuite ses fouilles, qui s'étendirent du fond de l'abri jusqu'à la limite du surplomb rocheux sur quasiment toute la longueur, de part et d'autre d'un énorme bloc qui délimita les parties ouest et est. La partie ouest, majoritairement fouillée, était essentiellement composée d'une " terrasse " que Girod, Massénat et Rivière ne fouillèrent intégralement que sur une vingtaine de mètres carrés, en laissant intactes les couches inférieure et moyenne sur tout le reste de l'espace que fouilla ensuite Peyrony. Le 


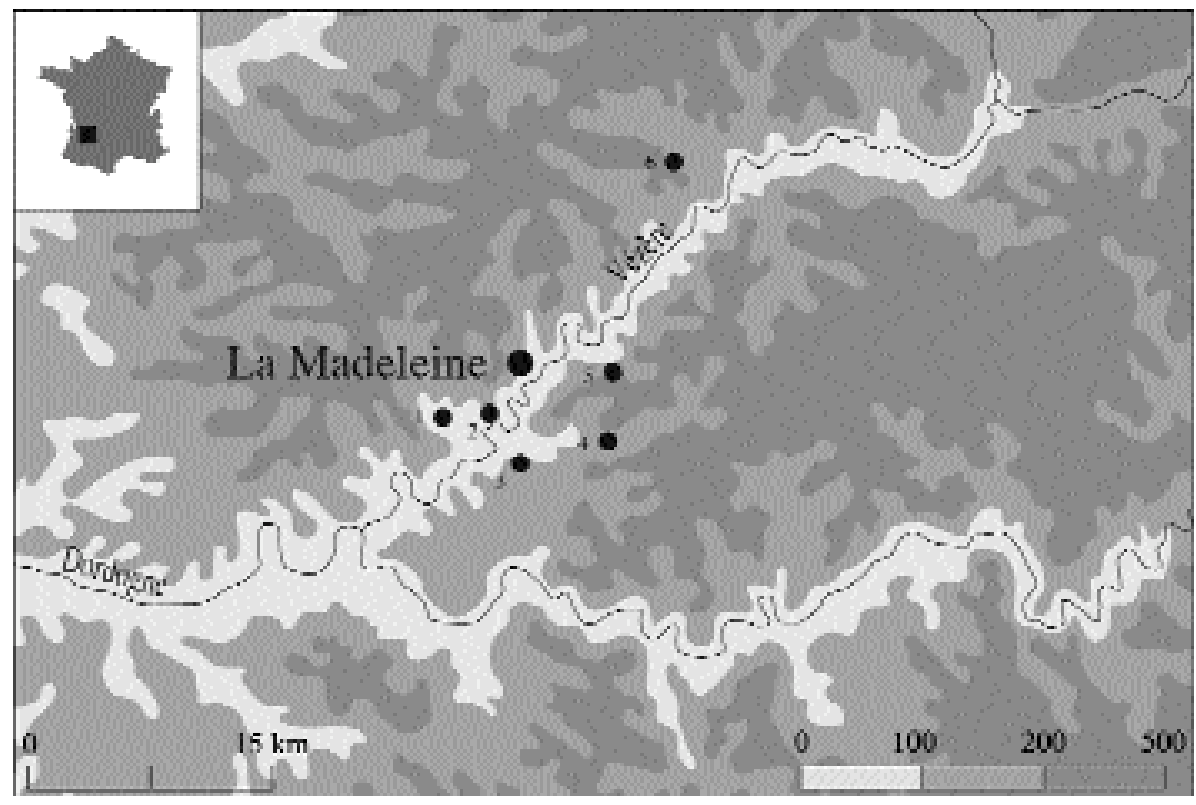

Figure 1 - Localisation du site de La Madeleine

(1. La Ferrassie,

2. Laugerie-Haute,

3. Abri Pataud,

4. Abris Lartet et Castanet,

5. Crabillat, 6. Badegoule).

Figure 1 - Location

of La Madeleine

(1. La Ferrassie,

2. Laugerie-Haute,

3. Abri Pataud,

4. Abris Lartet \& Castanet,

5. Crabillat,

6. Badegoule).

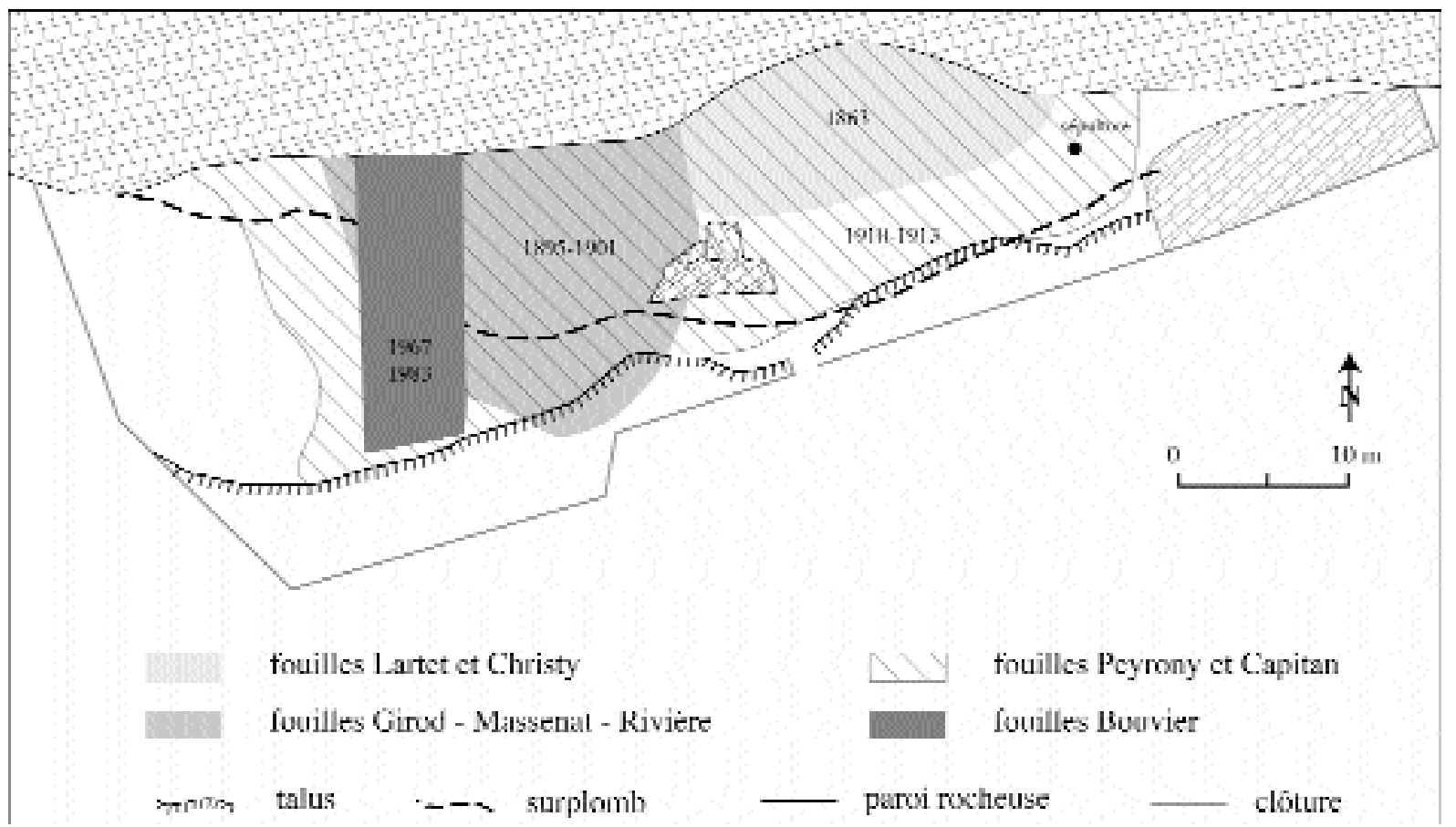

Figure 2 - Plan du site de la Madeleine et localisation des différentes fouilles.

Figure 2 - Map of La Madeleine and location of different excavations. 

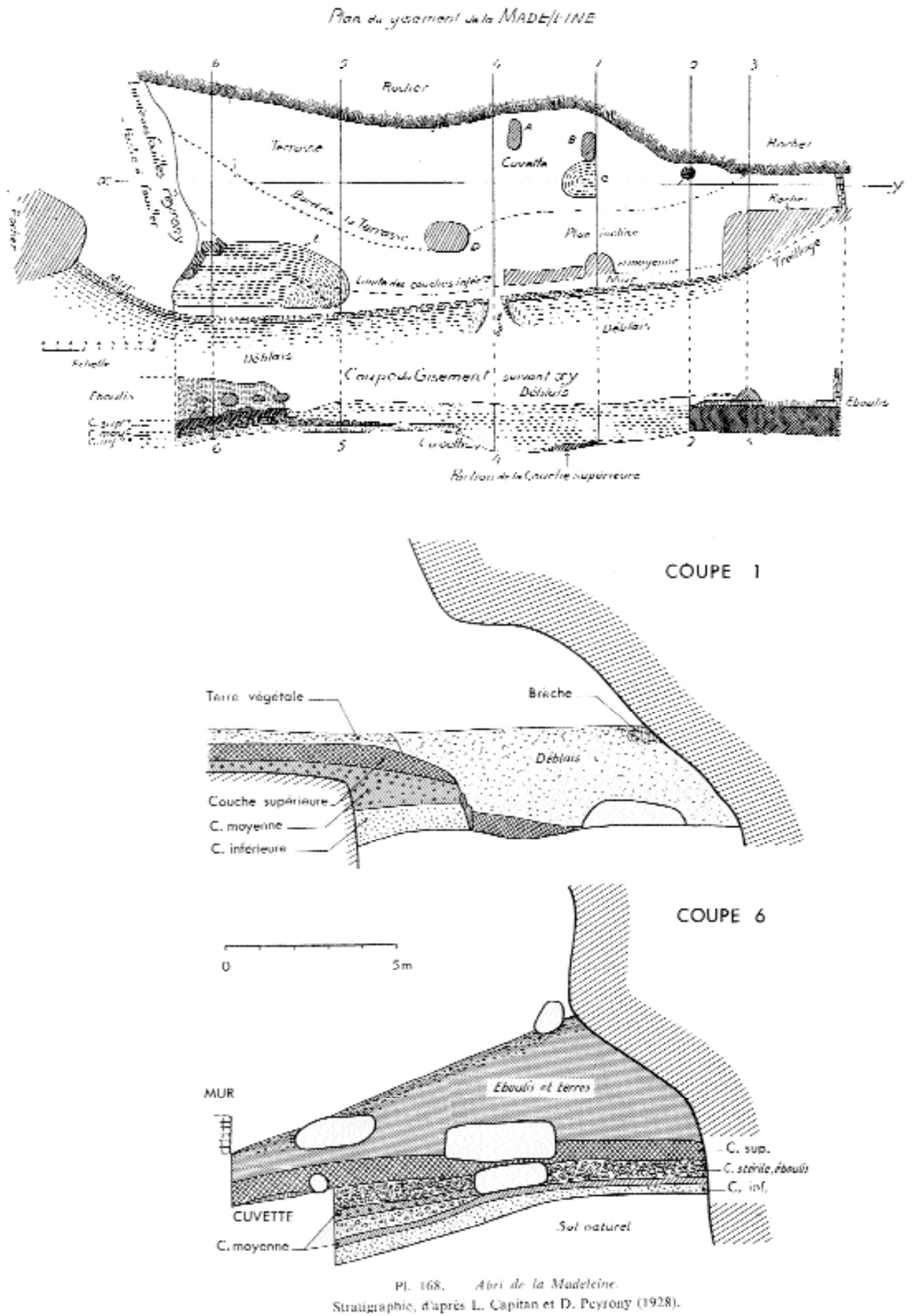

Figure 3a - Coupes stratigraphiques et plan de La Madeleine (Capitan \& Peyrony 1928).

Figure 3a - Stratigraphic sections and map of La Madeleine. 
secteur est était occupé en partie par une " grande cuvette " (cf. fig. 2), laissée intacte par Lartet et que Peyrony fouilla entièrement. II interrompit ses investigations en 1913 et ce n'est qu'en 1926 qu'il entreprit quelques travaux à l'est où il découvrit la sépulture d'un enfant (Bouvier 1972). La stratigraphie qu'il établit à partir du relevé de six coupes distingue trois couches sédimentaires qu'il assimila à trois ensembles archéologiques (fig. 3a). La couche inférieure, attribuée au Magdalénien IV, était constituée d'un " sable terreux d'inondation " dont l'épaisseur variait de 0,25 à 1 mètre (Capitan et Peyrony 1928, p. 17). La couche moyenne, d'une épaisseur variable non précisée, était à la base " de nature sableuse presque identique à l'inférieure " et dans sa partie supérieure " composée de petits éléments calcaires mélangés à du limon rouge brique, un peu argileux " (ibid., p. 63) ; elle renfermait le Magdalénien V. La couche supérieure, contenant le Magdalénien VI, était " brune, composée de terre et de nombreux éléments calcaires, gros et petits" et son épaisseur variait de 0,50 à plus d'1,50 mètre à l'est (ibid., p. 81). Dans ce vaste espace fouillé par D. Peyrony, les couches inférieure et moyenne étaient globalement réparties sur l'ensemble de l'abri à l'exception de la grande " cuvette" qui contenait uniquement la couche supérieure (ibid., p. 15). Celle-ci recouvrait en partie la couche moyenne à l'ouest et emplissait intégralement la grande " cuvette".

Les restes fauniques recueillis furent déterminés par É. Harlé (Mammifères) et M.E.F. Newton (Oiseaux) ${ }^{3}$. Une nouvelle détermination, doublée d'un dénombrement des restes d'ongulés, a été effectuée plus récemment (Madelaine 1989) ; elle vient compléter une étude paléontologique des restes de Lièvre variable (Donard 1982). L'industrie en matières dures d'origine animale a fait l'objet de plusieurs travaux (détail dans Bundgen 2002). Celle des séries Peyrony a servi de base à Henri Breuil pour la subdivision du Magdalénien moyen et supérieur en trois phases (Magdalénien IV, V, VI) (Breuil 1937). Les études de cette industrie sont nombreuses et elles ont porté sur les bâtons percés (Peltier 1992), les "sagaies " (Bouvier 1974 ; Delporte et al. 1988), les têtes de harpons (Julien 1982 et 1995), les foënes, hameçons et biseaux (Bellier et al. 1995 ; Averbouh et Cleyet-Merle 1995 ; Provenzano 1998) ainsi que les aiguilles à chas (Stordeur-Yedid 1979) et les crochets de propulseurs (Cattelain 1988) ${ }^{4}$. Plus récemment, certains restes d'ivoire de mammouth ont été analysés dans une perspective technique par C. Fradet (2005).

Les derniers travaux en date de J.-M. Bouvier (1967-1983), un sondage et des fouilles de 50 mètres carrés dans le secteur ouest (cf. fig. 2), visaient notamment à "affiner les distinctions stratigraphiques des couches sédimentaires et des niveaux archéologiques qu'elles contiennent... " (Bouvier 1977 , p. 64). L'étude de H. Laville mit en évidence trois ensembles stratigraphiques contenant 19 couches (fig. 3b). L'ensemble supérieur (couche A) correspondait à "une

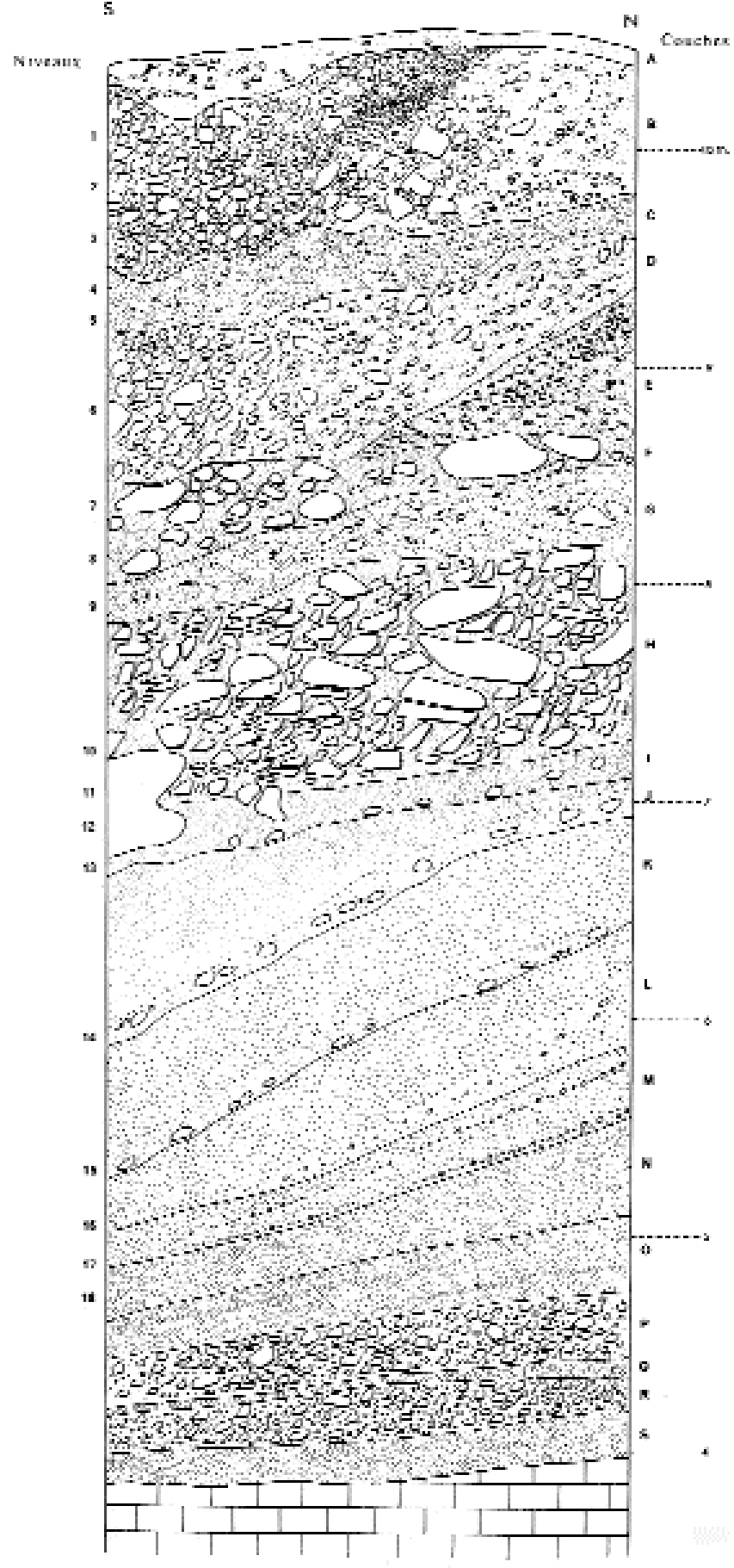

Figure $3 b$ - Coupe stratigraphique sagittale de la zone ouest fouillée par Bouvier (Laville 1975).

Figure 3b - Sagittal stratigraphic section of the western part Bouvier's excavations (Laville 1975).

(3) La liste des espèces figure dans la monographie (Capitan et Peyrony 1928, p. 62, 78, 100).

(4) Ces matériaux et ceux issus des fouilles Bouvier sont étudiés par L. Braem dans le cadre d'une thèse de doctorat en cours (Université de Provence). 
accumulation de castine sur 4,50 mètres d'épaisseur maximale". L'ensemble moyen (3, 5 mètres d'épaisseur), "d'origine exclusivement cryoclastique", contenait les vestiges des occupations du Magdalénien $\mathrm{V}$ et $\mathrm{VI}$ (couches $\mathrm{B}$ à $\mathrm{H}$ ). L'ensemble inférieur (3 mètres), "de nature essentiellement alluviale", comprenait les témoins d'occupation du Magdalénien IV et V (couches I à S) (Laville 1975, p. 326) ; il reposait sur le sol rocheux de l'abri. A partir des 18 niveaux archéologiques définis et renumérotés après 1975, Bouvier distingua cinq niveaux du Magdalénien IV (30 à 26), six niveaux du Magdalénien V (25 à 20) et sept niveaux du Magdalénien VI (19 à 13) (cf. fig. 3b). Notons que le sol rocheux de l'abri, atteint lors de ses fouilles, se situait " plusieurs mètres sous ce que D. Peyrony appelait le "sol naturel " du gisement." (ibid., p. 323). Bouvier n'attribua aucun de ces niveaux d'occupation à un Magdalénien antérieur au Magdalénien IV et confirma la seule présence du Magdalénien moyen et supérieur. Quatre des 18 niveaux archéologiques $(19,21,25,26)$ ont fait l'objet de datations radiocarbone (Bouvier 1973, 1977, 1979). Ainsi, le Magdalénien IV n'est daté que par le niveau le plus récent (26) - $13440+/-300$ BP (Ly 919) et aucun reste provenant des fouilles Peyrony n'a été daté. Une partie des vestiges fauniques issus des fouilles Bouvier a été déterminée par $F$. Delpech (Delpech 1983) et l'exploitation des bois de Renne a fait l'objet d'une première étude (Bonnissent 1993). Les pointes en matières dures d'origine animale ont fait l'objet d'une étude morphométrique (Bouvier 1974), puis l'ensemble de l'industrie fut inventoriée, sur des bases essentiellement typologiques, par B. Bundgen qui décompta 143 pièces (Bundgen 2002).

A l'issue de cette présentation succincte des différentes fouilles et études, il apparaît que les restes de Lièvre variable, manufacturés ou non, n'ont jamais fait l'objet d'une analyse approfondie. Ils n'ont jamais été comptabilisés, ni même triés dans l'optique d'une éventuelle identification de déchets d'industrie en ${ }^{5}{ }^{5}$. De plus, l'essentiel des restes de Lièvre exhumés lors des fouilles Peyrony provient de la base de la couche inférieure, horizon qui n'a jamais été mis en relation avec un ou plusieurs niveaux des fouilles Bouvier. Ceci est d'autant plus regrettable qu'aucun des niveaux des fouilles Bouvier ne contient une telle abondance de restes de Lièvre. C'est donc la question de la représentativité des collections qui se pose à présent.

\section{3 - Représentativité des séries Peyrony et Bouvier (Magdalénien IV)}

La question de la représentativité concerne d'une part, la totalité des restes fauniques et, d'autre part, les seuls restes de Lièvre variable. Dans quelle mesure les deux collections sont-elles représentatives, qualitativement (en termes spécifique et anatomique) et quantitativement de ce qui a été exhumé et de ce que contenait le site ? La représentativité des séries dépend avant tout des condi- tions de conservation, excellentes sur ce site. Elle dépend également des volumes et des surfaces fouillés ainsi que des méthodes de prélèvement, plus ou moins sélectif, qui ont pu être préjudiciables à la représentation des restes fauniques en général et du Lièvre en particulier. La représentativité des restes fauniques est également liée à l'histoire des collections, qui ont subi diverses " amputations" successives. Nous avons déjà montré, avec l'étude du Fou meau du Diable (Bourdeilles, Dordogne), que l'ancienneté des fouilles ne limite pas systématiquement et totalement la représentativité des assemblages fauniques ; chaque site est un cas particulier (Fontana 2001).

Qu'en est-il de la série Peyrony et tout d'abord de la collecte des restes fauniques? II est dit que "Toute la faune a été recueillie" (Capitan et Peyrony 1928, p. 62), ce dont on peut théoriquement douter en raison de l'absence de tamisage. Mais surtout, compte tenu de la surface fouillée (au moins 300 mètres carrés), du remplissage et de la densité du matériel, on imagine difficilement que la couche inférieure contenait seulement 2149 restes alors que les quatre niveaux inférieurs des fouilles limitées de Bouvier en ont livré près de 5400 (cf. infra). De plus, la rareté des restes de taxons de petite taille témoigne d'une récolte sélective, effectuée aux dépens des Poissons, des Oiseaux, des Micromammifères et, peut-être, des petits Camivores et des Lagomorphes. De la même façon, la représentation des parties squelettiques ${ }^{6}$ témoigne d'un tri sélectif au profit des parties aisément identifiables (dents, os entiers et extrémités d'os longs). Que penser alors de la représentativité des restes de Lièvre issus d'une telle collecte ? D. Peyrony fut frappé, sur la fouille même, par la grande quantité des restes de cet animal apparaissant sous la forme d'“ un lit d'ossements [...] de 4 à 5 centimètres d'épaisseur. " (ibid. p. 19) à la base de la couche inférieure. II affirme les avoir prélevés avec soin, ce que confiment la rareté des fractures récentes et la présence de quasiment toutes les parties du squelette (cf. infra). Cette concentration des restes a probablement rendu la collecte moins partielle que s'ils avaient été dispersés. De plus, s'il apparaît, à l'instar des restes des autres espèces, que les fragments de diaphyse n'ont pas été systématiquement récoltés, nous verrons que cela altère peu la représentation des parties du squelette. II semble donc que les choix de collecte de Peyrony n'ont pas agi en défaveur de la représentation des petits restes du Lièvre, que ce soit par rapport aux autres espèces ou en termes de représentation des parties squelettiques.

La représentativité de cette collection a-t-elle été altérée par la destination des restes de Lièvre ? Peyrony a vendu un grand nombre de pièces et le produit de ses fouilles fut dispersé en France comme à l'étranger. Nous avons constaté qu'aucun des musées français et allemands qui abritent certains objets issus de ces fouilles, ne possède plus de quelques restes de Lièvre. II est donc fort probable

(5) Denis Peyrony n'envisageait pas une origine anthropique pour ces restes qui lui semblaient plutôt être “... les reliefs des repas des grands rapaces nocturnes de cette époque. " (Capitan et Peyrony 1928, p. 20).

(6) Etude en cours du Magdalénien IV et V au Musée national de Préhistoire, L. Fontana. 
que la série Peyrony des restes de Lièvre conservée au Musée National de Préhistoire des Eyzies soit représentative de ce qui fut exhumé. C'est plutôt la perte des collections plus anciennes qui pose la question de la représentativité des restes de Lièvre de la série Peyrony. Néanmoins, puisque la base de la couche inférieure contenant ce "lit d'ossements ", n'avait été que très peu atteinte par les anciennes fouilles, à l'Ouest et au centre de l'abri, il est fort probable que ces travaux ne nous aient pas privés de nombreux restes de Lièvre.

En revanche, il semble que, lors de la conservation de la série Peyrony, une partie des restes de Lièvre ait été perdue après un tri par partie anatomique, avant qu'É. Donard ne réalise son étude paléontologique (cf. supra). En effet, il nous est immédiatement apparu que certains os faisaient défaut : quasiment tous ceux du squelette axial (vertèbres, côtes, sternum, os du crâne), tous les tarsiens à l'exception des calcanéums, tous les carpiens, les os sésamoïdes, les rotules, les phalanges II, III et les phalanges I antérieures. Ceci ne peut être interprété en termes de prélèvement de bas de pattes puisque les premières phalanges postérieures, les calcanéums et les métapodes sont présents. L'hypothèse de la récolte sélective (on ne collecte pas les plus petits os, difficiles à distinguer) n'est pas plus défendable : on aurait collecté les seules premières phalanges postérieures qui sont plus petites que les antérieures, les calcanéums et pas un seul astragale (à peine plus petit), pas un seul maxillaire ni une seule dent isolée mais de nombreux restes de microfaune ? De plus, la plupart de ces petits restes manquants ne sont en général pas fragmentés (comme les diaphyses dont Peyrony n'a pas prélevé ou conservé les petits fragments) dans les corpus connus. Encore une fois, la présence exclusive des premières phalanges postérieures constitue un argument fort pour penser que les restes de Lièvre ont été collectés dans leur intégralité, à l'exception des petits fragments de diaphyse. Dès lors, on peut imaginer qu'ils ont été triés et conditionnés par partie anatomique et qu'une partie des boîtes renfermant ces os a ensuite été égarée. Cette hypothèse est confirmée par la présence, parmi les restes fauniques, de deux petits blocs de terre, prélevés lors des fouilles Peyrony mais jamais " fouillés ", qui contenaient 31 restes de Lièvre : des sésamoïdes, deux os du tarse, des phalanges, une stemèbre, des côtes, des vertèbres. II semble donc que ces parties anatomiques étaient à l'origine présentes dans les dépôts archéologiques. Leur présence dans la collection Bouvier le confirme.

La question de la représentativité ne se pose pas dans les mêmes termes pour la série Bouvier. Le fait qu'un quart de la série faunique totale ne puisse être utilisée, car elle ne portait plus aucune mention des couches d'appartenance lors de son transfert au Musée national de Préhistoire des Eyzies en 2004-2005, n'a pas affecté nos données ${ }^{7}$. Nous savons en effet que cet échantillon ne contenait que de rares restes de Lièvre. Ceux de la collection Bouvier que nous avons étudiés sont issus de fouilles récentes dont les méthodes de prélèvement ne peuvent, en principe, avoir altéré la composition initiale de l'assemblage puisque tous les restes fauniques (y compris les fragments de diaphyse) furent recueillis et les sédiments tamisés à l'eau (maille inconnue). Pourtant, la représentation des parties squelettiques du Lièvre et la taille minimale des esquilles indiquent un biais qui témoigne que les plus petits restes de Lièvre n'ont pas été récoltés au tamisage et/ou au tri des refus de tamis. En ce qui concerne l'espace fouillé, le fait que la superficie ait été bien moindre que celle des fouilles Peyrony ne constitue pas, en soi, une limite à la représentativité de l'échantillon. C'est plutôt la combinaison de trois facteurs qui a pu intervenir dans la représentativité de la collection Bouvier (cf. fig. 2) : la localisation des fouilles à l'extrême ouest, la forme de l'espace fouillé qui correspondait davantage à une tranchée, la taille restreinte de la fouille comparée à celle de l'ensemble du site.

Pour conclure, les restes de Lièvre des séries Peyrony et Bouvier proviennent de secteurs différents qui n'avaient pas la même étendue ; ils n'ont pas été prélevés de la même façon et l'histoire de leur conservation ou de leur étude est différente. On peut déjà supposer que le manque de représentativité de la série Peyrony est bien identifié et qu'il se caractérise par une sous-représentation du nombre de restes, en majorité des fragments de diaphyse et de tout petits os. En revanche, on ignore dans quelle mesure le fait que les vestiges proviennent d'une surface limitée, excentrée par rapport aux fouilles antérieures, altère la représentativité des restes issus des fouilles récentes. C'est l'étude détaillée qui peut contribuer à répondre à cette question.

\section{2 - LA PART DU LIÈVRE VARIABLE ET LES MODALITÉS DE SON ACQUISITION}

Le fait que la quasi-totalité des restes de Lièvre de la série Peyrony provient de la couche inférieure attribuée au Magdalénien IV, est confirmé : 752 des 762 restes appartiennent à cette couche. II en est de même pour la série Bouvier dont 283 restes proviennent des couches (26 à 29) du Magdalénien IV et 50 des couches (25 à 21) du Magdalénien $\mathrm{V}$ (tabl. 1). Examinons maintenant en détail la question de la part du Lièvre, à partir de notre échantillon de 1035 restes composé des deux collections du Magdalénien IV (tabl. 2).

\section{1 - Le Lièvre, le Renne et le Cheval}

Dans les deux séries, 99 \% des restes identifiés appartiennent à trois espèces dont la part diffère d'une collection à l'autre. Les restes de Lièvre représentent, dans la série Bouvier et d'après nos derniers décomptes, 5,3\% des restes appartenant aux trois premiers gibiers. Cette part du Lièvre est très proche de celle indiquée $(7 \%)$ par $F$. Delpech (1983) dans l'étude d'un échantillon de 1264 restes déterminés, pour les quatre niveaux du Magdalénien IV, issu du produit des fouilles 1968-1978

(7) Ce problème n'a pu être résolu en dépit du fait que les restes en question portent la mention de leur carré ainsi qu'un numéro d'objet car l'accès aux carnets de fouille nous a été refusé par le fouilleur. 


\begin{tabular}{|l|r|}
\hline Fouilles Peyrony & NR \\
\hline C. inférieure (M. IV) & 752 \\
\hline C. moyenne (M. V) & 10 \\
\hline Total & $\mathbf{7 6 2}$ \\
\hline & \multicolumn{1}{|r|}{} \\
\hline liouilles Bouvier & NR \\
\hline C..29 à 26 (M. IV) & 283 \\
\hline C..25 a 21 (M. V) & 50 \\
\hline Total & $\mathbf{3 3 3}$ \\
\hline
\end{tabular}

Tableau 1 - Décompte des restes de Lièvre variable dans le Magdalénien IV et $V$ des fouilles Peyrony et Bouvier.

Table 1 - Count of Arctic Hare remains in the Magdalenian $I V$ and $V$ levels (Peyrony and Bouvier's excavations).

\begin{tabular}{|l|r|}
\hline Fouilles Peyrony & NR \\
\hline Couche inférieure & $\mathbf{7 5 2}$ \\
\hline \multicolumn{2}{|c|}{} \\
\hline Fouilles Bouvier & NR \\
\hline Niveau 29 & 2 \\
\hline Niveau 28 & 143 \\
\hline Niveau 27 & 105 \\
\hline Niveau 26 & 33 \\
\hline Total \\
\hline \multicolumn{2}{|l}{} \\
\hline Total & $\mathbf{2 8 3}$ \\
\hline
\end{tabular}

Tableau 2 - Décompte détaillé des restes de Lièvre variable dans le Magdalénien IV des fouilles Peyrony et Bouvier.

Table 2 - Detailed count of Arctic Hare remains in the Magdalenian IV (Peyrony and Bouvier's excavations).

\begin{tabular}{|l|r|r|}
\hline Taxons & NR & \% NR \\
\hline Rangifer farundus & 873 & 40,62 \\
\hline Lepus timidus & 752 & 34,99 \\
\hline Equus cahallus & 503 & 23,41 \\
\hline Bos/Bison & 14 & 0,65 \\
\hline Rupicapra rupicapra & 7 & 0,33 \\
\hline Total & $\mathbf{2 1 4 9}$ & \\
\hline
\end{tabular}

Tableau 4 - Représentation des taxons identifiés pour la couche inférieure du Magdalénien IV des fouilles Peyrony.

Table 4 - Identified taxa representation in the Peyrony's excavations Magdalenian IV levels.

\begin{tabular}{|l|c|c|c|c|}
\hline & \multicolumn{2}{|c|}{ Coll. Bouvier } & \multicolumn{2}{c|}{ Coll. Peyrony } \\
\hline Espèces & NR & $\%$ NR & NR & $\%$ NR \\
\hline Rangifer tarandus & 4560 & 84,87 & 873 & 41,02 \\
\hline Lquus cuhallus & 530 & 9,86 & 503 & 23,64 \\
\hline Lepus timidus & 283 & 5,27 & 752 & 35,34 \\
\hline Total & $\mathbf{5 3 7 3}$ & & $\mathbf{2 1 2 8}$ & \\
\hline
\end{tabular}

Tableau 5 - Part relative des trois premiers gibiers dans les couches du Magdalénien IV des fouilles Bouvier et Peyrony.

Table 5 - Representation of the three main game in Magdalenian IV levels (Bouvier and Peyrony's excavations).

\begin{tabular}{|c|c|c|c|c|c|c|}
\hline Delpech, 1983 & C 29 & C 28 & C 27 & C 26 & Total & $\% \mathrm{NR}$ \\
\hline Rangifer tarandus & 20 & 163 & 506 & 247 & 936 & 74,05 \\
\hline Bos/Bison & & & 1 & 5 & 6 & 0,47 \\
\hline Rupicapra rupicalpra & & 1 & & & 1 & 0,08 \\
\hline Capra ibex & & 1 & 1 & & 2 & 0,16 \\
\hline Fquaus caballus & 2 & 18 & 70 & 141 & 231 & 18,28 \\
\hline Lepus sp. & 8 & 39 & 16 & 25 & 88 & 6,96 \\
\hline Total & 30 & 222 & 594 & 418 & 1264 & \\
\hline Estimation L. liontana & C:29 & C: 28 & C. 27 & $C: 26$ & Total & $\%$ NR \\
\hline Rangifer tarandus & & 2000 & 2500 & 60 & 4560 & 84,87 \\
\hline Equas cahollus & & 200 & 300 & 30 & 530 & 9,86 \\
\hline Lepus timidus & 2 & 143 & 105 & 33 & 283 & 5,27 \\
\hline Total & 2 & 2.343 & 2905 & 123 & 5373 & \\
\hline
\end{tabular}

Tableau 3 - Représentation des taxons identifiés pour les niveaux du Magdalénien IV (C. 26 à 30) des fouilles Bouvier (Delpech 1983 et Fontana en cours).

Table 3 - Identified taxa representation in the Bouvier's excavations Magdalenian IV levels (26 to 30) - Delpech 1983 ; Fontana on going. 


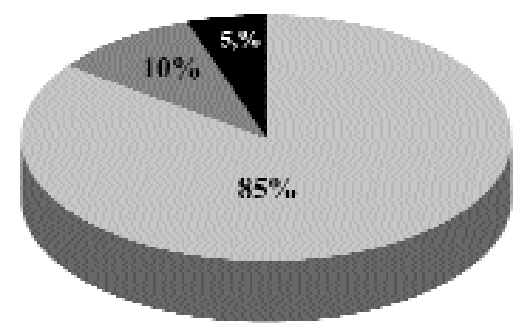

Rangifer tarandus $\mathbf{E}$ Equas caballus

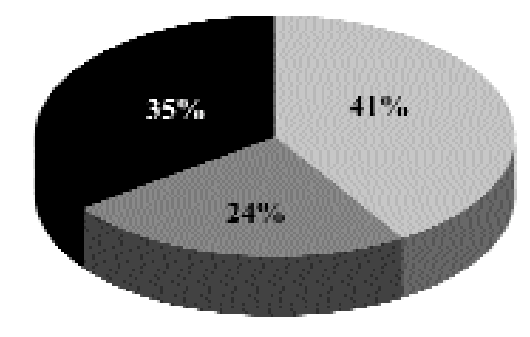

I.epus timidus
Figure 4 - Part relative des trois principaux gibiers dans les couches du Magdalénien IV des fouilles Peyrony et Bouvier.

Figure 4 - Representation of the three main game in the Peyrony \& Bouvier Magdalenian IV levels excavations. (tabl. 3). Si le nombre de restes de Lièvre (283) est aujourd'hui quatre fois plus important, c'est au sein d'une collection qui compte également quatre fois plus de restes (5 373). Dans cette série récente, le Lièvre est le troisiè me gibier, après le Cheval et le Renne ${ }^{8}$. II semble donc nettement moins bien représenté que dans la série Peyrony où il est le second gibier après le Renne et devant le Cheval, au sein d'un spectre bien plus diversifié (tabl. 4). Néanmoins, la place réelle du Lièvre par rapport aux deux autres gibiers est, dans la série Peyrony, très difficile à estimer (tabl. 5 et fig. 4). Cette série compte 2,5 fois moins de restes que la collection Bouvier, en dépit de la grande surface fouillée et en raison d'une collecte sélective mais trois fois plus de restes de Lièvre qui représentent $35 \%$ de l'ensemble. Les restes de Renne et/ou de Cheval ont été eux aussi partiellement collectés et on ignore donc si la part de chacun des trois gibiers est représentative ou bien si une des trois espèces est sous ou sur-représentée. La comparaison avec les chiffres de la série Bouvier est là encore instructive (cf. tabl. 5 et fig. 4). La différence est pour le moins surprenante puisque la part du Renne dans la série Peyrony est de $40 \%$ (cinq fois moins de restes) contre $80 \%$ dans la série Bouvier. On peut imaginer que le nombre initial de restes de Renne était bien plus important car il est peu probable que le Renne représentait $80 \%$ des restes dans 50 mètres carrés situés à l'ouest et seulement $40 \%$ sur la plus grande partie de l'abri anciennement fouillé. On connaît la grande dispersion des vestiges de Renne de la collection Peyrony et elle peut en grande partie expliquer cette faible part du Renne. Quoi qu'il en soit, si la représentation du Renne dans la collection Peyrony fut de $80 \%$ comme dans les fouilles Bouvier, cela signifie que ce gibier est bien plus sous-représenté que le Lièvre. Ceci était notre hypothèse de départ en raison de la concentration des restes de Lièvre, du soin que Peyrony a apporté à leur collecte, comme le confirme la présence de certains petits os (infiniment plus petits que ceux récoltés pour le Renne), et de leur moindre dispersion. II est donc fort probable que la part du Renne soit en réalité bien plus importante que celle du Lièvre. De la même façon, il est possible que la représentation du Cheval obéisse à une logique identique à celle du Renne, ses restes ayant probablement été collectés de la même façon. La part du Lièvre est donc surestimée dans la série Peyrony, aux dépens de celle du Cheval et du Renne, et le spectre faunique, moins diversifié.

Retenons que, dans la série Bouvier, la part de 5,3\%, qui place le Lièvre au rang de troisième gibier, est représentative de l'espace fouillé alors que celle de $35 \%$ pour la série Peyrony, issue d'une collecte probablement sélective au profit du Lièvre (second gibier), ne l'est pas. La part du Lièvre à La Madeleine se situe donc probablement entre 6 et $25 \%$.

\section{2. - Origine des restes de Lièvre variable et modalités d'acquisition du gibier}

L'exploitation du petit gibier, qu'il s'agisse de lagomorphes, de petits camivores, d'oiseaux ou de poissons, ne laisse pas systématiquement d'indices tels que des traces de silex, des fractures et une représentation des parties squelettiques caractéristiques, ou encore une surreprésentation des individus adultes. De plus, la probabilité d'identifier des témoins d'une exploitation du petit gibier décroit avec le nombre de restes, ce qui pose problème dans le cas des petites séries, majoritaires en Europe occidentale (Fontana 2004). Ceci est d'autant plus gênant dans les contextes d'abris et de grottes où peuvent se trouver des restes de Lièvre issus du repas de certains rapaces et camivores. C'est un ensemble d'indices, voire des témoignages indirects, qui permet de démontrer le caractère anthropique de l'origine de restes de lièvres, à condition de disposer d'une série conséquente. Cette question ne se pose pas à La Madeleine, puisque, une fois n'est pas coutume, son exploitation a laissé de nombreux indices et notamment des traces de silex identifiées sur 46 restes osseux. Elles traduisent divers types d'actions liées

(8) Les décomptes présentés pour le Renne excluent les bois, en cours de tri. 
Carré |C. Partic anatomique

Type de trace

Idcntification

Figures

\begin{tabular}{|c|c|c|c|c|c|c|c|c|c|}
\hline K 17 & 261 & I lumărus & Diaphyse & Raclisye & Icongitudinales & antêrièure & lines, trüs lines & $\because ?$ & lix. 12.1 \\
\hline $\mathrm{L} 27$ & $26 \mathrm{r}$ & Iíbia & Diaphysc & Raclage & longitudinales & latćrale & très fincs & $9 ?$ & fig. 13.4 \\
\hline $\mathrm{K} 17$ & 26F & Radius gauche & Din-cpi.dist & Raclage & longitudinales & antéricure & fincs & $? ?$ & fig. 12.1 \\
\hline M 16 & 26 & 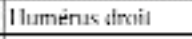 & Tipiph. dist & Découme & perpenuliculaires: & lateriale & Irès: problinnies: & désumicalaliım & \\
\hline K 17 & 26 & Ratius gatuche & 1 Dia-čpi_prox. & Díxлия & oxhliy̨us & liatürále & prolisnibes & dhosarnisstiuxn? & lig. 112.1 \\
\hline $\begin{array}{l}\text { L } 17 \\
\text { L } 26\end{array}$ & $\begin{array}{c}26 \mathrm{~F} \\
26\end{array}$ & $\begin{array}{l}\text { Radius gauclic } \\
\text { Humcrus droit }\end{array}$ & $\begin{array}{l}\text { Diaphyse } \\
\text { Diaphyse }\end{array}$ & $\begin{array}{l}\text { Licoupe } \\
\text { Dbcoupe }\end{array}$ & $\begin{array}{l}\text { obliques } \\
\text { obliques }\end{array}$ & $\begin{array}{l}\text { latćcalc } \\
\text { latcralc }\end{array}$ & $\begin{array}{l}\text { fincs, tres fines } \\
\text { profondes }\end{array}$ & $\begin{array}{l}\text { decarnisation } \\
\text { desarticulntion }\end{array}$ & fig .10 .2 \\
\hline K 16 & 27 & Tihia gauche & I Diaplyy:e & Rasilisge \& Jis: & Iang. \& abliques & liatéralle & lines & dicismisolinxi? & liي. 1.3 .5 \\
\hline $\begin{array}{l}18 \\
\text { M } 19 \\
\end{array}$ & \begin{tabular}{l|}
27 \\
27 \\
\end{tabular} & $\begin{array}{l}\text { rémur droit } \\
\text { Tibia gauche }\end{array}$ & $\begin{array}{l}\text { Dia-cpi.dist. } \\
\text { Epiph. dist. }\end{array}$ & $\begin{array}{l}\text { Rainurage } \\
\text { Rainurnge }\end{array}$ & $\begin{array}{l}\text { longitudinales } \\
\text { longitudinalcs }\end{array}$ & $\begin{array}{l}\text { latćrale \& ant. } \\
\text { antéricure }\end{array}$ & & $\begin{array}{l}\text { matrice à aiguilles } \\
\text { matrice ả aiguilles }\end{array}$ & $\begin{array}{l}\text { fig. } 14.1 \\
\text { fig. } 14.4\end{array}$ \\
\hline $\mathrm{K} 18$ & 27 & Indêterminte & Diaphyse & Raclaype & Ianypitudinales & indèterminête & Irès fines: & $? ?$ & tịp. 17. $\mathrm{K}$ \\
\hline M 17 & 27 & l'ëmuar ůrovil & 1 Diaphyse & Raclisge & Icongitudirnales & lasterriale & lines & $\because ?$ & lig. 13.2 \\
\hline H 18 & 27 & Mctat. IV droit & Diaphys: & Decoupe & obliques & lanćrale & profonds: & dípouillenent? & fig. II \\
\hline 117 & 28 & I IIna & Гpiph. dist. & Ravlaype & lonypitudinales & inierne & lines, très tines & $\%$ & fijp. 12.5 \\
\hline 116 & 28 & Radies druil & 1 Dia épri.prox. & Raclasye & Iongiludinales & antérieure & Irés lintes & $\because ?$ & lig. 12.2 \\
\hline $\begin{array}{l}\text { HJ } 18 \\
\text { I } 18\end{array}$ & $\begin{array}{l}28 \\
28 \\
\end{array}$ & $\begin{array}{l}\text { Indcterminć } \\
\text { Indéterminé }\end{array}$ & $\begin{array}{l}\text { Diapliys: } \\
\text { Diaphyse }\end{array}$ & $\begin{array}{l}\text { Rainurage } \\
\text { Raclage }\end{array}$ & $\begin{array}{l}\text { longitudinales } \\
\text { longitudinales }\end{array}$ & $\begin{array}{l}\text { indsermince } \\
\text { indeterminte }\end{array}$ & trçs fincs & $\begin{array}{l}\text { baguctic } \\
? ?\end{array}$ & $\begin{array}{l}\text { fig. } 14.5 \\
\text { fig. } 13.7\end{array}$ \\
\hline 118 & 28 & Mếtatıırsien III & Diil-ępi-prox. & Sectionnement & perpendiculaire & circulaire & Irès profiande & miltrice is tuhes: & fig. 16 \\
\hline 118 & 28 & Tihiz ulruì & I Diaphyse & Racliage & Ionyiludirnales & lastêriale & lines, Irês lines: & $\because$ & lig. 13.3 \\
\hline$J 16$ & 28 & Hunnctus gauche & Dia-cpidisis & Dccoupe & perp. \& obliques & latcrale \& ant. & profondes & dcsant. \& dscann.? & \\
\hline \multicolumn{2}{|c|}{ "Sandi. 29" } & Fèmer itruit & Diaphyse & Raclaste & lanypitudininiles & intérieıse & Iries fines; & $?$ & fịr. 17.1 \\
\hline \multicolumn{10}{|c|}{ Vouillex Peyrony - N : 25} \\
\hline \multirow{19}{*}{\multicolumn{2}{|c|}{$\begin{array}{l}\text { Cousche } \\
\text { infericurs }\end{array}$}} & $\begin{array}{l}\text { Mandiluale droile } \\
\text { Huménus droit }\end{array}$ & $\begin{array}{l}\text { Be. horizont. } \\
\text { Epiph. dist. }\end{array}$ & $\begin{array}{l}\text { Decoupe } \\
\text { Decoupe }\end{array}$ & $\begin{array}{l}\text { obliques } \\
\text { perpendiculaires }\end{array}$ & $\begin{array}{l}\text { exicme } \\
\text { latcicale \& ant. }\end{array}$ & $\begin{array}{l}\text { fincs } \\
\text { trçs profondes }\end{array}$ & $\begin{array}{l}\text { depouillenent } \\
\text { désarticulation }\end{array}$ & $\begin{array}{l}\text { fig. } 8 \\
\text { fig. } 8.2\end{array}$ \\
\hline & & Ilumeñus diroit & Гjpiph. dist. & Décourpe & perpendiculaine; & latenile 8 int. & profíniles & désarticalatiom & \\
\hline & & Radius dnil & I Diaphyse & \multicolumn{3}{|c|}{ 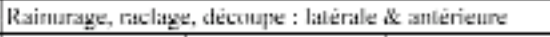 } & usus types & In. í aiguilles \& décamm. & lig. 14.2 \\
\hline & & $\begin{array}{l}\text { Radius droit } \\
\text { Radius droit }\end{array}$ & $\begin{array}{l}\text { Dia-cpi-dist. } \\
\text { Lia-cpi.prox. }\end{array}$ & $\begin{array}{l}\text { Dicoupe } \\
\text { Découpe }\end{array}$ & $\begin{array}{l}\text { obliques } \\
\text { perp. \& obliques }\end{array}$ & $\begin{array}{l}\text { latćcale } \\
\text { antéricure }\end{array}$ & $\begin{array}{l}\text { ues profondes } \\
\text { fincs, profondes }\end{array}$ & $\begin{array}{l}\text { desarticulation } \\
\text { decarnisntion? }\end{array}$ & fig. 9.3 \\
\hline & & Rathiss dmit & Diup-ëpi.dist, & Décourpe & whliques: & latêrile & lines, très fines & décarnisation & 1ị: 10.4 \\
\hline & & Radius dnuil & Diaphyse & Racliage & Icnyiludirnales & liaterratle \& anl. & Lrés: lintes & $\%$ & \\
\hline & & $\begin{array}{l}\text { Radius droit } \\
\text { Radius gauche }\end{array}$ & $\begin{array}{l}\text { Diaphlyse } \\
\text { Diaphyse }\end{array}$ & $\begin{array}{l}\text { Raclage } \\
\text { Raclage }\end{array}$ & $\begin{array}{l}\text { longitudinales } \\
\text { longitudinales }\end{array}$ & $\begin{array}{l}\text { latćralc } \\
\text { latéralc \& post }\end{array}$ & $\begin{array}{l}\text { utes fincs } \\
\text { fincs }\end{array}$ & $\begin{array}{l}? ? \\
? ? \\
\end{array}$ & fig. 12.3 \\
\hline & & Rondius gyuche & Diil-épi,dist. & Discoupe & obliques & laterale & fines & décamisntiven & \\
\hline & & Ratiis giuche & Dia épi. prux. & Décolupe & perperuliculaires: & liateriale & Irés problindies & décarnis:aliven? & \\
\hline & & $\begin{array}{l}\text { Radius gauche } \\
\text { Ulna gauche }\end{array}$ & $\begin{array}{l}\text { Diaphyse } \\
\text { Epipl. prox. }\end{array}$ & $\begin{array}{l}\text { Dícxupe } \\
\text { Découps }\end{array}$ & $\begin{array}{l}\text { oxhliyues } \\
\text { perpendiculaircs }\end{array}$ & $\begin{array}{l}\text { lațíralc } \\
\text { latécrale }\end{array}$ & $\begin{array}{l}\text { fincs } \\
\text { fincs }\end{array}$ & $\begin{array}{l}\text { dicsanisalixi } \\
\text { dicsarticulation }\end{array}$ & \\
\hline & & Fémur dirvit & Diiphyse & Raclaste \& déc: & longit \& perp. & laterale & fines, profondes & 77 \& décrmisgtivn? & fige 13,6 \\
\hline & & Coxial ulronil & Ischiver prixx. & Décсuаре & perp. $\alpha$ abliques: & exieme & Lusus types: & decarnisalivan & lig. 112.3 \\
\hline & & $\begin{array}{l}\text { Conal urwil } \\
\text { Coxal droit }\end{array}$ & $\begin{array}{l}\text { Ischiox prox. } \\
\text { Ischion prox. }\end{array}$ & $\begin{array}{l}\text { Déculupe } \\
\text { Découpe }\end{array}$ & $\begin{array}{l}\text { obliques } \\
\text { obliques }\end{array}$ & $\begin{array}{l}\text { exteme } \\
\text { extenc }\end{array}$ & $\begin{array}{l}\text { fincs } \\
\text { fincs }\end{array}$ & $\begin{array}{l}\text { dicarnisstixin } \\
\text { decarnisation }\end{array}$ & \\
\hline & & Tibias druit & Diaphyse & Raclagre & longhitudinales & laténale & fines & $? ?$ & \\
\hline & & Tihia dirvil & 1 Diat épi.dlisi. & Rainuer. $\alpha$ dëc: & Ianyil. \& remp. & ant. $\mathbb{N}$ puxt. & lines & In. à aiyuilles $\mathcal{N}$ détarm. & lig. $14.3,9.4$ \\
\hline & & $\begin{array}{l}\text { Tíbia gasuchic } \\
\text { Tibia gauche }\end{array}$ & $\begin{array}{l}\text { Dia-ţ̧i. prox. } \\
\text { Epipla. dist. }\end{array}$ & $\begin{array}{l}\text { Raclage } \\
\text { Découpc }\end{array}$ & $\begin{array}{l}\text { Ingitudinales } \\
\text { perpendiculaircs }\end{array}$ & $\begin{array}{l}\text { latćrale } \\
\text { postécicure }\end{array}$ & $\begin{array}{l}\text { fincs, lin's fincs } \\
\text { profoades }\end{array}$ & $\begin{array}{l}? " \\
\text { désarticulation }\end{array}$ & \\
\hline & & Tibia druit & Diaphyse & Decoupe & perpendiculaines & latenale & fines & décamisativen & \\
\hline & & Tihiz unvoil & 1 Diaphyse & Décourpe & perp. $N$ abliques: & latléritle & lines, proliandes & decarnisalinn & \\
\hline & & Calcancumn droit & Epqiph. prox. & Dixolupe & perpondiculairss & antéru-posL. & profonds & disarticulation & fig. $8,9.1$ \\
\hline
\end{tabular}

Tableau 6 - Inventaire des traces d'outils lithiques identifiées sur les 46 restes de Lièvre variable des fouilles Bouvier (couches 26 à 28) et Peyrony (couche inférieure).

Table 6 - Inventory of lithic marks identified on 46 Arctic Hare bones (Bouvier's excavations: levels 26 to 28) ; Peyrony's excavations: lower level). 


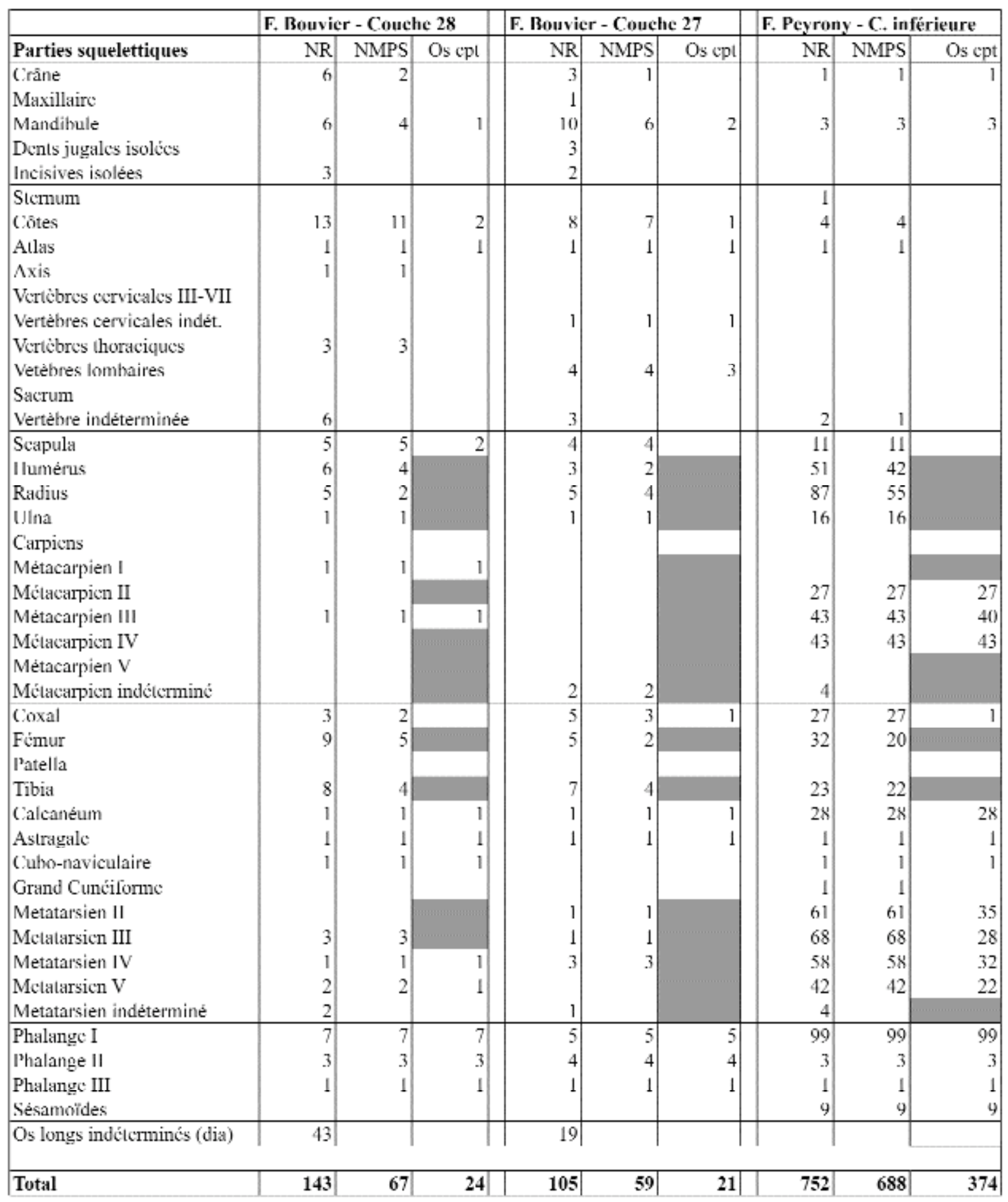

Tableau 7 - Décompte global des parties squelettiques de Lièvre variable des fouilles Bouvier (couches 27 et 28) et Peyrony (couche inférieure) (en grisé : aucun os long complet).

Table 7 - Global representation of Arctic Hare skeletal parts (Bouvier's excavations: levels 27 and 28) ; Peyrony's excavations: lower level). 
au traitement des carcasses, à la fabrication de supports et/ou d'objets (tabl. 6 et cf. infra) ${ }^{9}$. Seuls deux restes portent des traces laissées par des carnivores. Plus de $95 \%{ }^{10}$ des individus représentés sont des lièvres adultes, ce qui renforce le premier diagnostic : les levreaux n'ont pas été capturés ou, s'ils l'ont été, n'ont pas été rapportés sur le site. Le troisième indice réside dans la représentation des parties squelettiques qui correspond, en tous points, à ce que l'on connaît des accumulations anthropiques (Hockett 1991, 1992, 1994 ; Morel et Müller 1997 ; Speth 2000 ; Hockett \& Haws 2002 ; Fontana 2003 ; Cochard 2004) : présence de toutes les parties du squelette, proportions équivalentes des membres antérieur et postérieur, abondance des os des membres et des ceintures, forte représentation des métapodes et notamment des métatarsiens, sous-représentation du squelette axial et forte représenta tion de la mandibule par rapport au crâne (tabl. 7). Enfin, la fracturation des restes de la collection Bouvier présente les caractéristiques relatives à une accumulation anthropique puisque les os longs complets sont rares (aucun en C. 27, 4 métapodes en C. 28 , cf. tabl. 7 ), comme sur de nombreux sites (Hockett 1992 ; Morel et Müller 1997 ; Haws 2003). En revanche, les cylindres diaphysaires (notamment de tibias), qui constituent, pour certains, une preuve d'extraction de la moelle (par exemple Cochard 2004 ; Hockett 1991 et 1995 ; Morel et Müller 1997 ; Perez Ripoll 1993 ; Schmitt 1990), sont rarissimes dans la collection Bouvier (aucun en C. 28, 2 tibias en C. 27) et peu nombreux dans la collection Peyrony (deux humérus, deux radius et quatre tibias pour trois fois plus de restes). Les fragments d'os longs cylindriques, abondants, sont plutôt des diaphyses dotées d'une des deux extrémités. Elles témoignent d'un autre type de cassure, par flexion, qui est également documenté dans les assemblages anthropiques (Hockett \& Bicho 2000 ; Perez Ripoll 2002). D'autres morphologies de bords de fracture traduiraient la fracturation par percussion (cf. infra). Enfin, l'intervention d'autres prédateurs (camivores) est limitée et identifiable grâce aux traces figurant sur deux diaphyses de radius (série Bouvier). II ne fait donc aucun doute que les lièvres de La Madeleine ont été la proie des Magdaléniens.

A quel(s) moment(s) de l'année ont-ils été chassés ou piégés ? On peut émettre une hypothèse à partir des trois seuls restes immatures, qui indiquent la présence d'individus âgés de neuf mois. Si l'on considère l'existence, à partir des données actuelles de l'éthologie, d'une ou plusieurs périodes de reproduction entre les mois de mai et août, et si ces trois levreaux ont été capturés aux mêmes moments que les adultes, alors les captures auraient eu lieu entre les mois de février et mai. On ne peut cependant exclure, en raison de la rareté des restes de levreaux, la possibilité de captures à plusieurs moments de l'année et ce d'autant que les rennes ont été chassés en automne, en hiver et au printemps, voire en été (Bouchud 1966 ; Fontana en cours). Enfin, la présence de quasiment tous les éléments du squelette atteste que les dépouilles furent rapportées entières, comme dans les quelques autres sites connus (Cochard 2004 ; Fontana 2003 ; Morel et Müller 1997 ; Müller dans Bullinger et al. 2006). Des lièvres adultes furent donc capturés et rapportés entiers au site où le traitement de leur carcasse s'est déroulé selon des modalités qui restent maintenant à identifier.

\section{3 - CARACTÉRISTIQUES DE L'EXPLOITATION DU LIÈVRE}

L'enjeu de l'étude est de déterminer la nature de l'exploitation du Lièvre variable : fut-elle ciblée sur un produit ou fut-elle multiple? Cette question est fondamentale si l'on veut comprendre la place du Lièvre dans l'économie et donc identifier le caractère, impératif ou opportuniste, de son acquisition. Pour cela, il faut identifier les produits qui furent prélevés, consommés et utilisés puis caractériser leur exploitation (systématique ou anecdotique, plus ou moins intense). Mais la mise en évidence du prélèvement et de la consommation de certains produits est parfois problématique et discerner la part de chacun d'entre eux l'est bien davantage, pour des raisons d'ordre méthodologique, comme nous allons l'illustrer maintenant.

\section{1 - La représentation des parties du squelette}

L'analyse des parties du squelette témoigne, directement et indirectement, des modalités d'apport et surtout de rejet des carcasses, au même titre que l'étude de la fragmentation et des traces mais d'une façon différente. Précisons que la représentation des parties squelettiques est calculée en \% des parties observées (\% PO) ${ }^{11}$. La représentation des parties squelettiques du Lièvre de la série Peyrony présente une particularité déjà évoquée : les plus petits os sont absents ou rarissimes et témoignent d'une collecte sélective (tabl. 8 ; fig. 5). On note également la présence de tous les os longs et des ceintures, dans des proportions diverses qu'il s'agit d'interpréter (cf. fig. 5). La forte représentation des métapodes, notamment des métatarsiens, peut s'expliquer par le fait que ce sont, parmi les os longs, ceux qui sont le moins fragmentés : seuls trois des 113 métacarpiens ne sont pas entiers et $51 \%$ des 116 métatarsiens le sont, alors que parmi tous les autres os longs, aucun n'est entier.

(9) Tous les restes osseux et dentaires de Lepus timidus ont été examinés à la loupe binoculaire.

(10) Trois restes témoignent de la présence de rares individus plus jeunes : une extrémité proximale d'humérus juste soudée dans la collection Peyrony et deux corps immatures de vertèbres cervicale et lombaire dans la collection Bouvier.

(11) Le \% PO est calculé de la façon suivante: (NMPS/NMlf x Nb parties squelettiques dans le squelette) $x$ 100. L'utilisation de ce paramètre permet de savoir comment est représentée chaque partie anatomique par rapport à ce qu'elle devrait être si les carcasses étaient entières. On rapporte donc le nombre minimal de chaque partie anatomique ( $D+G+i n d e ́ t$.$) au nombre atten-$ du qui est estimé en fonction du nombre minimal de lièvres (voir Fontana 2003). 


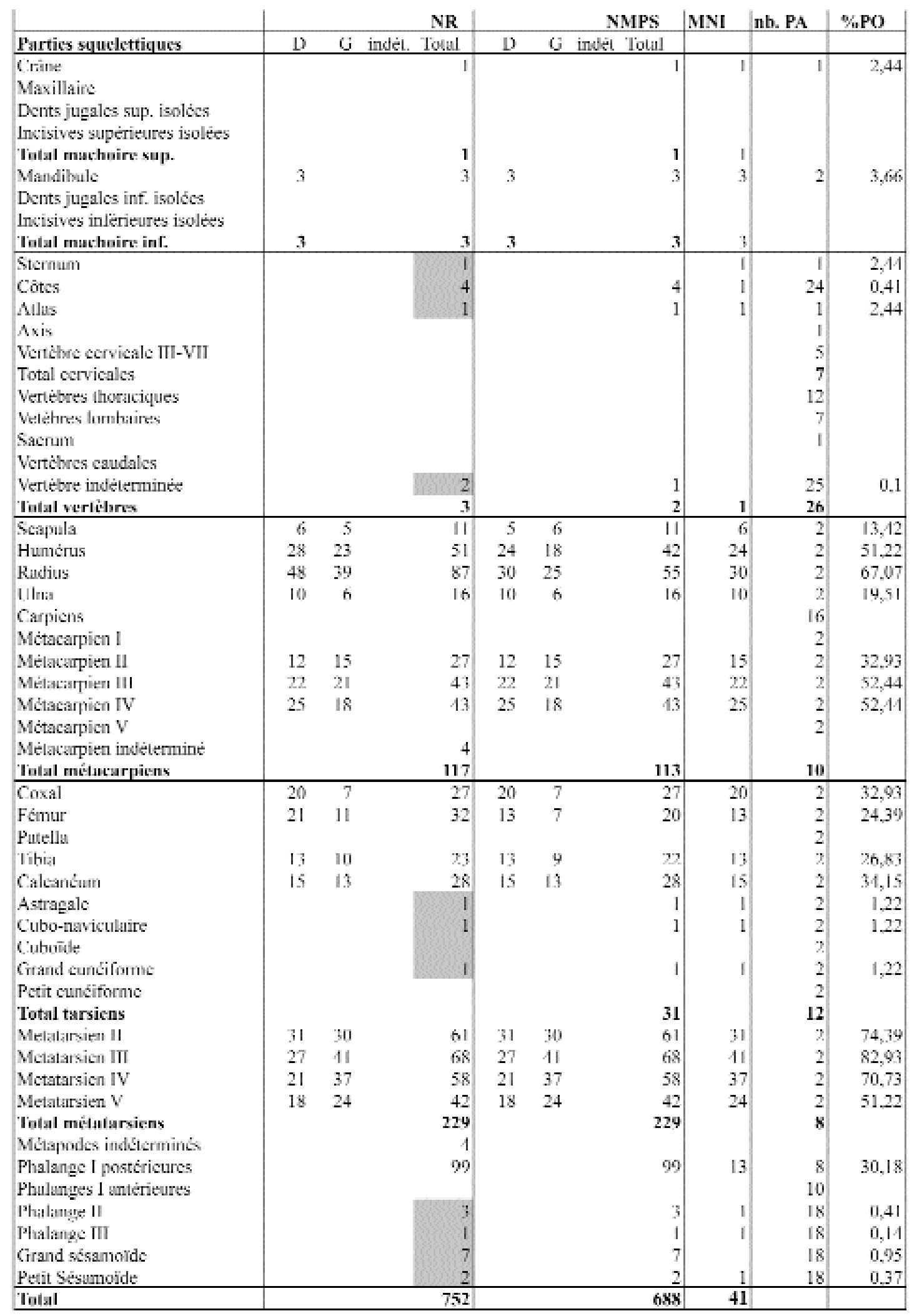

Tableau 8 - Représentation des parties squelettiques du Lièvre variable de la couche inférieure des fouilles Peyrony (en grisé : restes exhumés lors de la fouille d'un petit bloc de sédiment en 2006).

Table 8 - Arctic Hare skeletal parts representation in the Peyrony's lower level. 


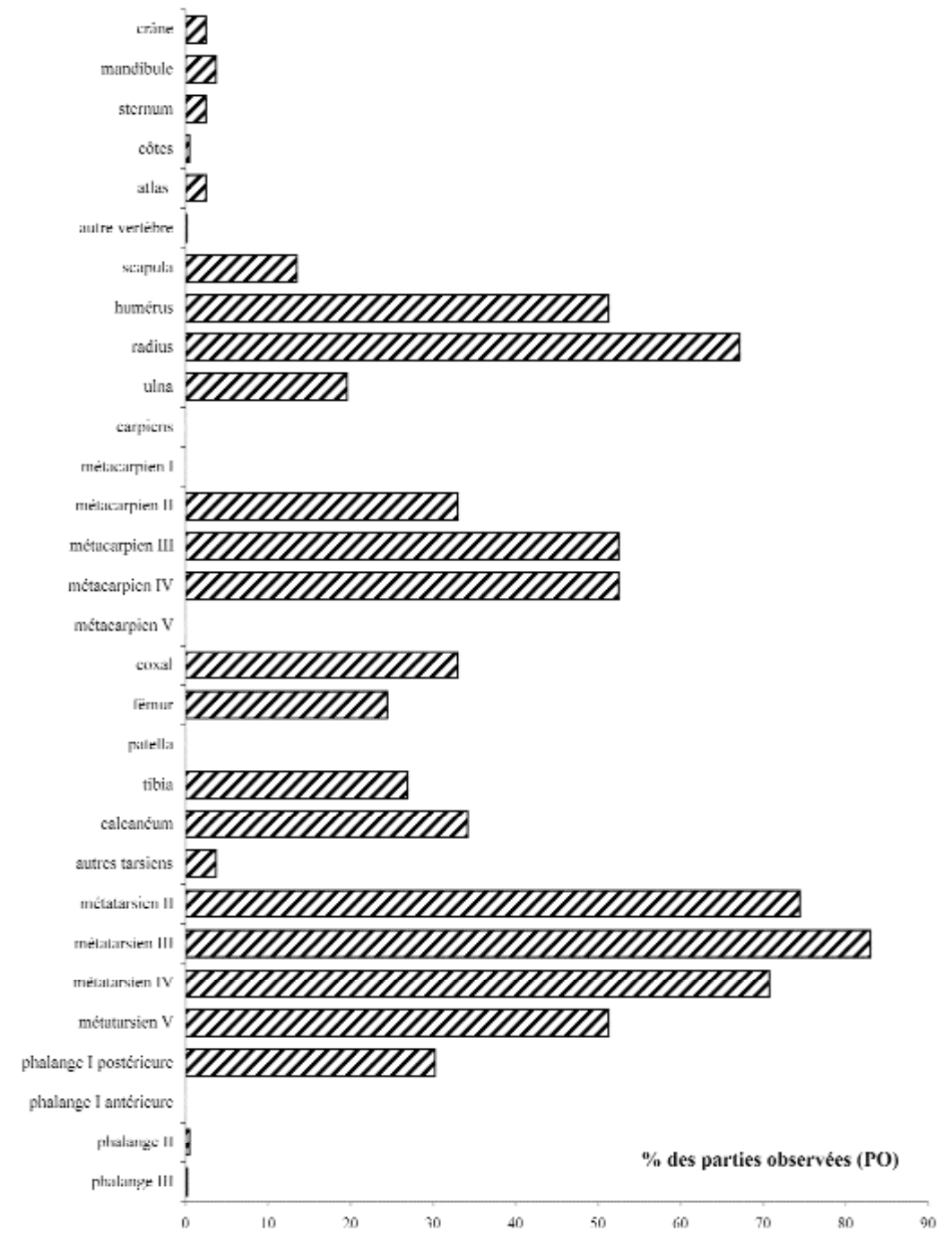

Figure 5 - Représentation des parties squelettiques du Lièvre variable de la couche inférieure (fouilles Peyrony) du Magdalénien IV (NMPS : 688).

Figure 5 - Skeletal part frequencies of Arctic Hare in the Magdalenian IV lower level (Peyrony's excavations) MNE: 688.

Davantage fragmentés, et donc moins bien collectés, ces derniers sont sous-représentés par rapport aux métapodes. La seconde caractéristique est l'homogénéité des représentations par segment anatomique. Trois des quatre métatarsiens (II, III, IV) sont représentés entre 65 et $80 \%$ et les os du reste du membre postérieur (calcanéum, tibia, fémur et coxal) se situent tous entre 25 et $35 \%$. II en est de même pour le membre antérieur si l'on admet que la faible représentation de la scapula et de l'ulna, os plus fragiles et plus fragmentés, dont seule l'articulation proximale est majoritairement consenée, peut résulter de la collecte sélective. Leur plus forte représentation dans la série Bouvier confirme cette hypothèse. Ces représentations assez homogènes par segment sont plutôt inattendues pour une série issue de fouilles anciennes. II semblerait que, si les diaphyses fragmentées d'os longs n'ont pas été collectées, les épiphyses (avec ou sans fragment de diaphyse) l'aient été, ce qui renforce la représentativité de cet échantillon et montre que, dans ce cas, ne pas collecter les fragments de diaphyse n'altère pas ou très peu la représentation relative des os longs (à l'exception des métapodes, très peu fragmentés). Cela témoigne à nou- veau du potentiel réel des collections fauniques anciennes, notamment du point de vue de la représentativité anatomique, comme nous l'avons déjà démontré pour les fouilles Peyrony du Fourneau du diable, à partir des restes de Renne (cf. supra). Enfin, cette homogénéité n'est pas spécifique de La Madeleine (excepté le fait qu'elle concerne des fouilles anciennes) puisqu'elle transparaît également dans les seules données détaillées de Gazel, de Champréveyres et de Bois Ragot (Morel et Müller 1997 ; Fontana 2003 ; Cochard 2004). Le point fondamental est qu'une telle homogénéité de la représentation des parties squelettiques de Lièvre est supérieure à celle des grands gibiers, notamment le Renne, dans la grande majorité des sites magdaléniens (par exemple Castel 1999 ; Fontana 1998 et 1999 ; Morel et Müller 1997 ; Bridault 1996). Doit-on y voir un rejet solidaire par segment, avec ou sans prélèvement systématique de viande crue? Si tel était probablement le cas pour des sites où les os sont très peu fracturés (comme à Gazel), il est plus difficile de l'envisager pour les séries de restes très fracturés, comme celles de La Madeleine. On s'attendrait également à la présence de connexions radius-ulna-humérus et tibia- 


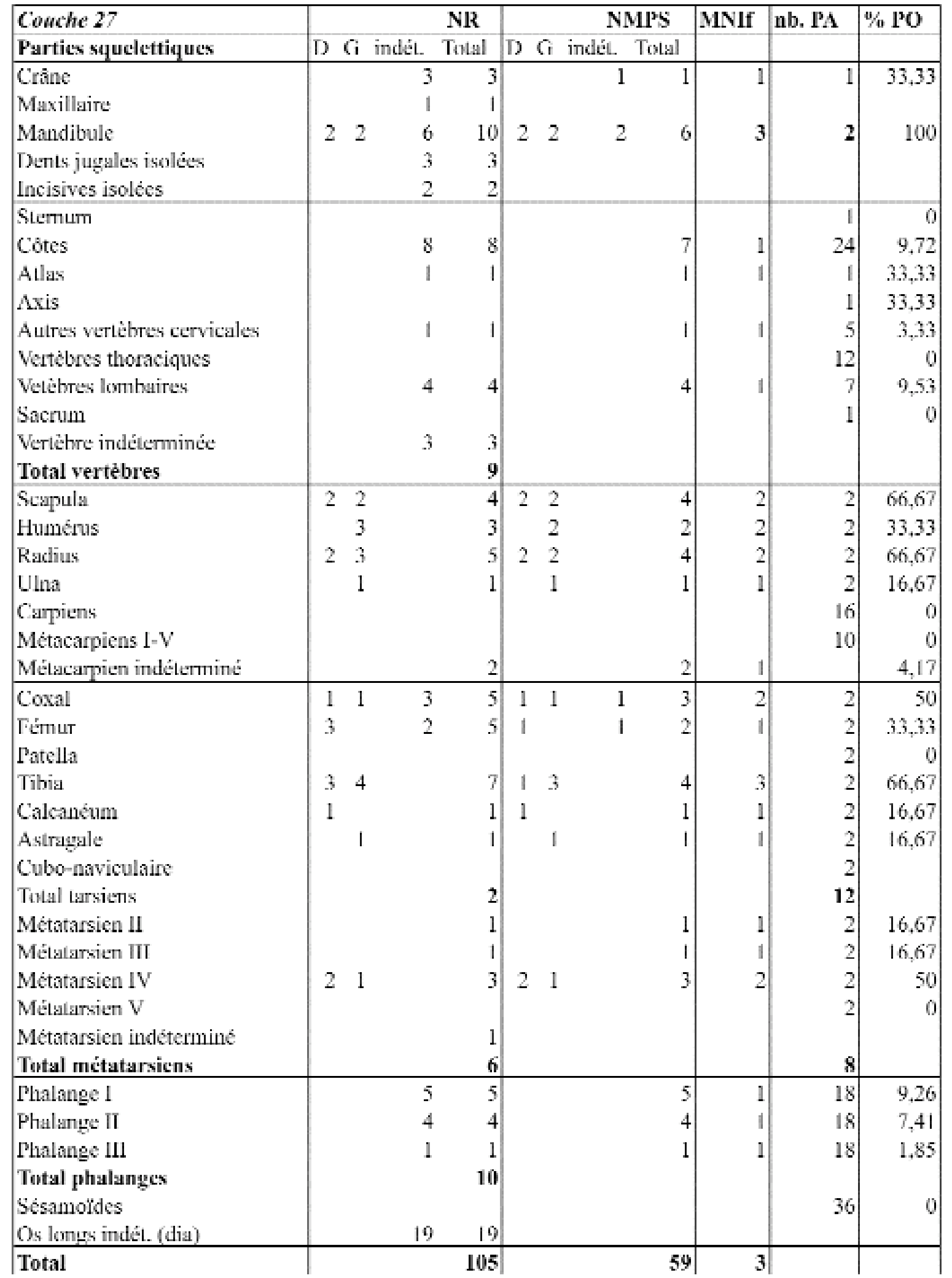

Tableau 9 - Représentation des parties squelettiques du Lièvre variable de la couche 27 des fouilles Bouvier.

Table 9 - Arctic Hare skeletal parts representation in Bouvier's excavations level 27. 


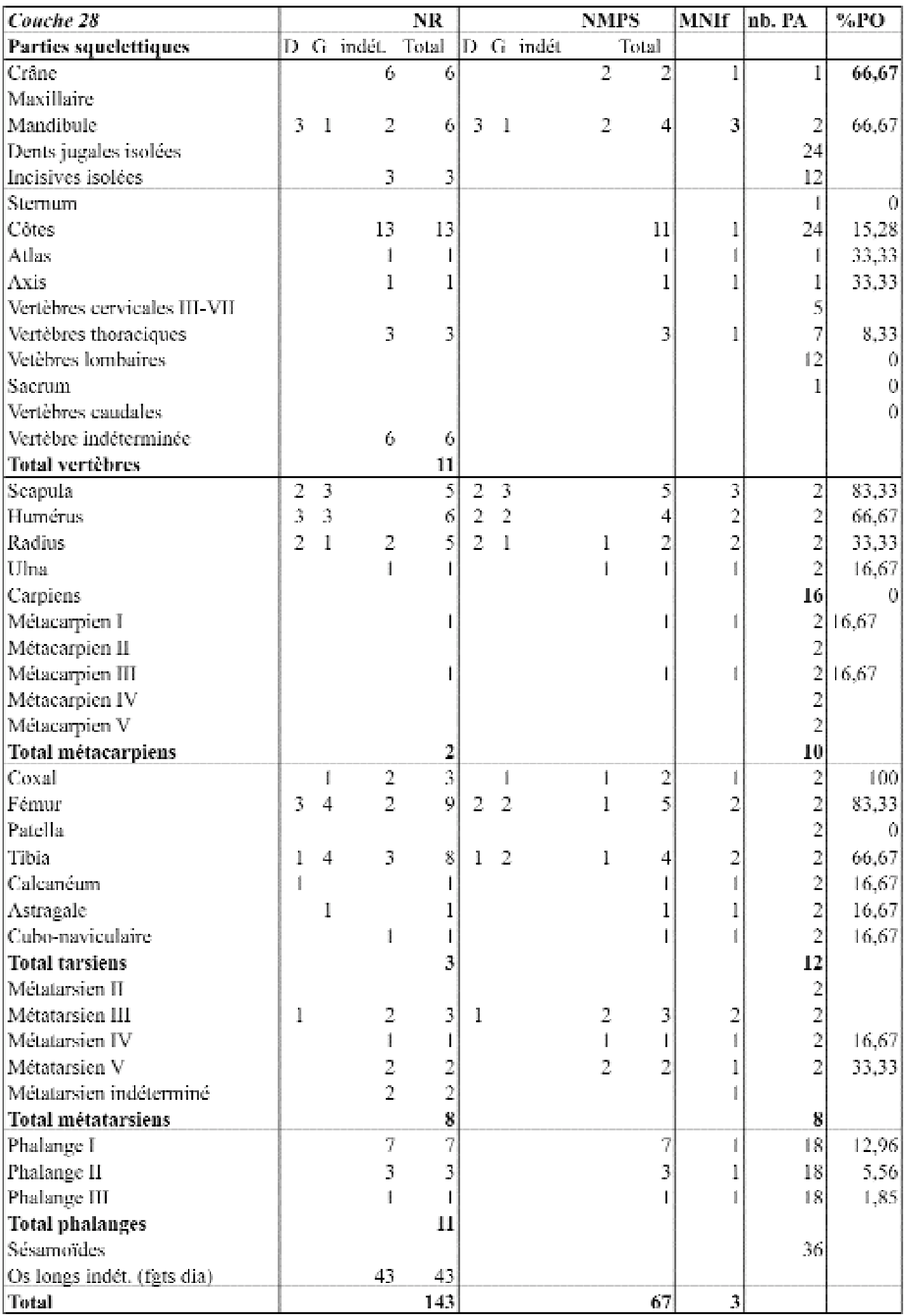

Tableau 10 - Représentation des parties squelettiques du Lièvre variable de la couche 28 des fouilles Bouvier.

Table 10 - Arctic Hare skeletal parts representation in Bouvier's excavations level 28. 


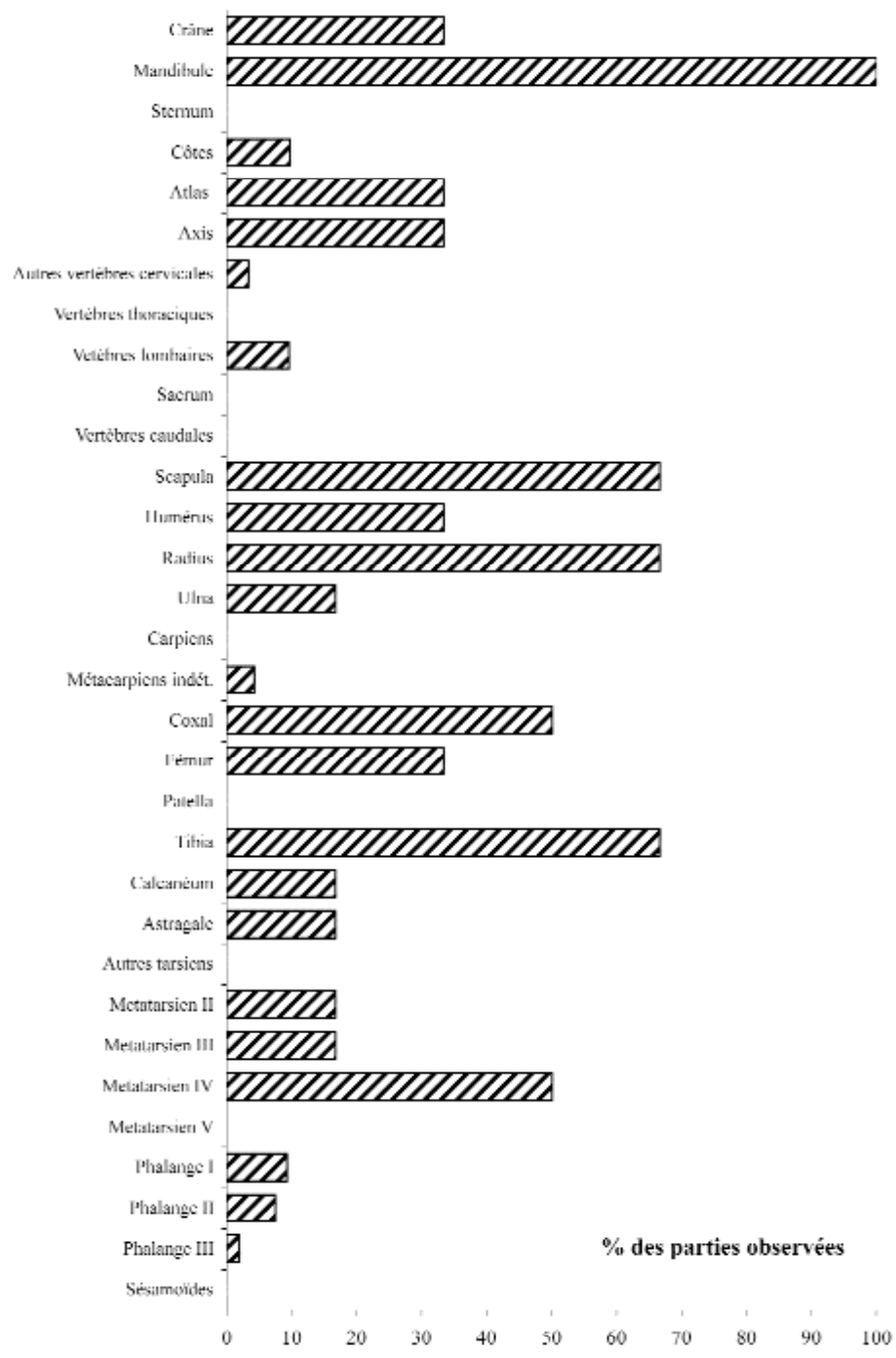

Figure 6 - Représentation des parties squelettiques du Lièvre variable de la couche 27 (fouilles Bouvier) du Magdalénien IV (NMPS : 59).

Figure 6 - Skeletal part frequencies of Arctic Hare in the Magdalenian IV level 27 (Bouvier's excavations) - MNE: 59.

fémur, ce qui n'est pas le cas à La Madeleine où aucune connexion n'a été notée mais aussi à Gazel et à Bois-Ragot où elles ne concernent que le rachis et l'autopode (Fontana 2003 ; Cochard 2004). On pourrait alors envisager une variante à l'hypothèse du rejet solidaire : celle d'un rejet localisé qui expliquerait l'homogénéité de la représentation des os de Lièvre appartenant aux mêmes segments. D'ailleurs, Peyrony avait noté que les restes de lièvre étaient concentrés, non seulement à la base de la couche inférieure mais également dans la cuvette centrale (cf. supra). Néanmoins, les segments anatomiques (membres antérieur et postérieur, rachis, crâne) ne sont pas représentés de façon semblable, notamment en raison de la sur-représentation des métapodes. Doit-on considérer leur taux de représentation comme plus ou moins représentatif de celui de tous les os longs ? On ne peut évacuer la possibilité d'une représentation différentielle des membres antérieur et postérieur qui serait due à un prélèvement ou une fragmentation particulière d'un ou plusieurs os. Néanmoins, il est fort probable que le radius, le mieux représenté des autres os longs l'ait été initialement dans des proportions voisines à celle des métatarsiens : il fut lui aussi moins fracturé et il ne fut probablement pas sujet à une exploitation alimentaire. De plus, la comparaison avec la série Bouvier tend à confirmer l'hypothèse d'une représentation homogène de tous les segments anatomiques.

Les deux couches analysées de la série Bouvier (C. 27 et 28) qui comptent le plus de restes apparaissent comme une version "corrigée " de la représentation de la série ancienne (tabl. 9 et 10). En effet, si la représentation des os appartenant aux mêmes segments est également plutôt homogène, le nombre d'os représentés au-delà de $50 \%$ passe de quatre à huit (fig. 6 et 7). Ceci renforce l'hypothèse selon laquelle la représentation globale des os longs tendrait vers $70 \%$. D'ailleurs, le décalage entre la représentation du membre antérieur et celle du membre postérieur est bien moindre, cette fois au profit du membre postérieur. Enfin, la très forte représentation de la mandibule, qui correspond davantage aux rares données existantes, et des restes crâniens, témoigne bel et bien du rejet des têtes sur le site. 


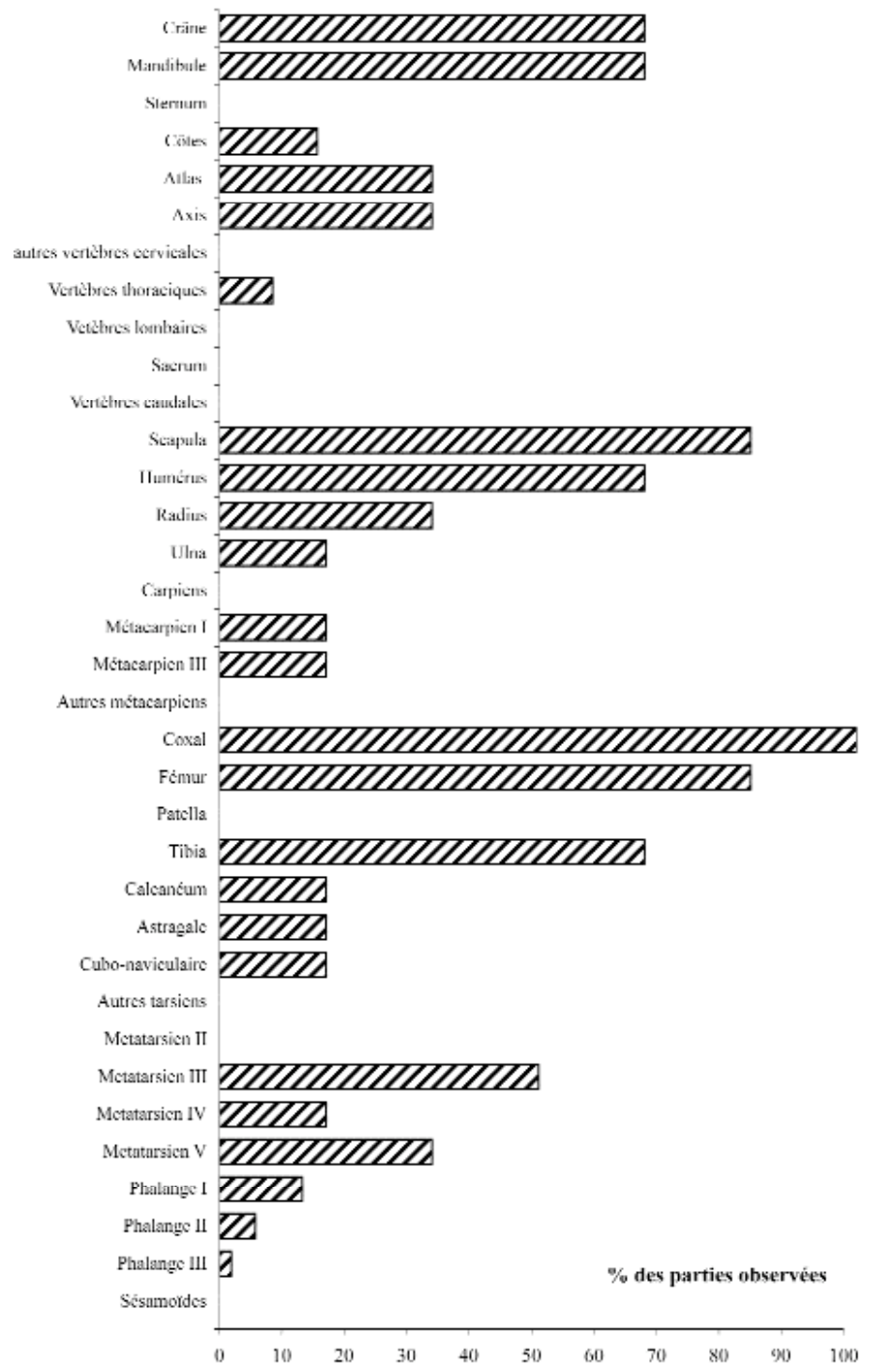

Figure 7 - Représentation des parties squelettiques du Lièvre variable de la couche 28 (fouilles Bouvier) du Magdalénien IV (NMPS : 67).

Figure 7 - Skeletal part frequencies of Arctic Hare in the Magdalenian IV level 28 (Bouvier's excavations) - MNE: 67.

Au terme de l'examen des deux collections, on constate que les principales caractéristiques de la représentation des parties squelettiques du Lièvre sont globalement identiques d'une série à l'autre et identiques à celles recensées dans de nombreux sites. Cela témoigne, selon nous, non pas d'un rejet solidaire des segments, puisque les os ont été fracturés pour récupérer la moelle, mais d'un rejet bien plus localisé que celui des restes de grands herbivores, de surcroit plus fragmentés. Pour conclure sur le traitement de la carcasse, sur l'importance de la consommation de viande crue et/ou cuite et sur le prélèvement de la fourrure (suggéré par l'absence des patellas et des vertèbres caudales), il faut à présent analyser les autres données relatives aux traces présentes sur les os.

\section{2 - Les traces}

L'observation des surfaces osseuses à la loupe binoculaire a été facilitée car les traces d'altération produites par les sédiments, l'eau ou encore les végétaux ne concernent que quelques restes. L'identification de traces anthropiques permet de savoir si la carcasse a été désarticulée et de quelle manière, si on a extrait la moelle, prélevé la peau et la viande (crue ou cuite) et de quelle façon, ou si on a fabriqué des objets à partir de certains restes. A La Madeleine, des " stries de découpe " (25), des traces de raclage (18), de sectionnement (1) et de rainurage (4) figurent sur 46 restes, certains d'entre eux portant plusieurs types de traces différentes (tabl. 11 cf. tabl. 6 et fig. 8). Les "stries de découpe" témoignent de la désarticulation, de la décarnisation ou encore de l'enlèvement de la peau.

Les stries liées à la désarticulation, profondes, obliques ou transversales sont observables sur les extrémités distales de cinq humérus, sur un radius, une ulna, un tibia et un calcanéum (fig. 9). Ce sont des traces banales, autant du point de vue de leur localisation (cf. fig. 8) que de leur répartition (Aura et al. 2002 ; Tagliacozzo \& Fiore 1998). Elles attestent de la séparation, au moyen d'un outil tranchant, des portions humérus/radius-ulna et tibia/basipode. Si d'autres segments ont été désarticulés, ils l'ont été soit de la même manière, mais sans laisser de traces, soit à la main. 


\begin{tabular}{|c|c|c|c|c|c|c|c|}
\hline Lièvre variable & NR & NRD & NMPS & NMIf & NR Tr: & NR racl. & Déchets \\
\hline C. 26 Bouvier & 33 & 32 & 20 & 3 & 4 & 3 & 0 \\
\hline (.. 27 l3ouvier & 105 & 86 & 63 & 3 & 2 & 3 & 2 \\
\hline C. 28 Bouvier & 143 & 100 & 74 & 3 & 1 & 4 & 2 \\
\hline C.29 13ouvier & 2 & 2 & 2 & 1 & 0 & 1 & 0 \\
\hline C. inférieure l'eyrony & 752 & 752 & 696 & 41 & 18 & 7 & 2 \\
\hline Total deux séries & 1035 & 972 & 855 & 41 & 25 & 18 & 6 \\
\hline
\end{tabular}

Tableau 11 - Décompte des restes de Lièvre variable portant des traces.

Table 11 - Account of Arctic Hare remains bearing anthropic marks.
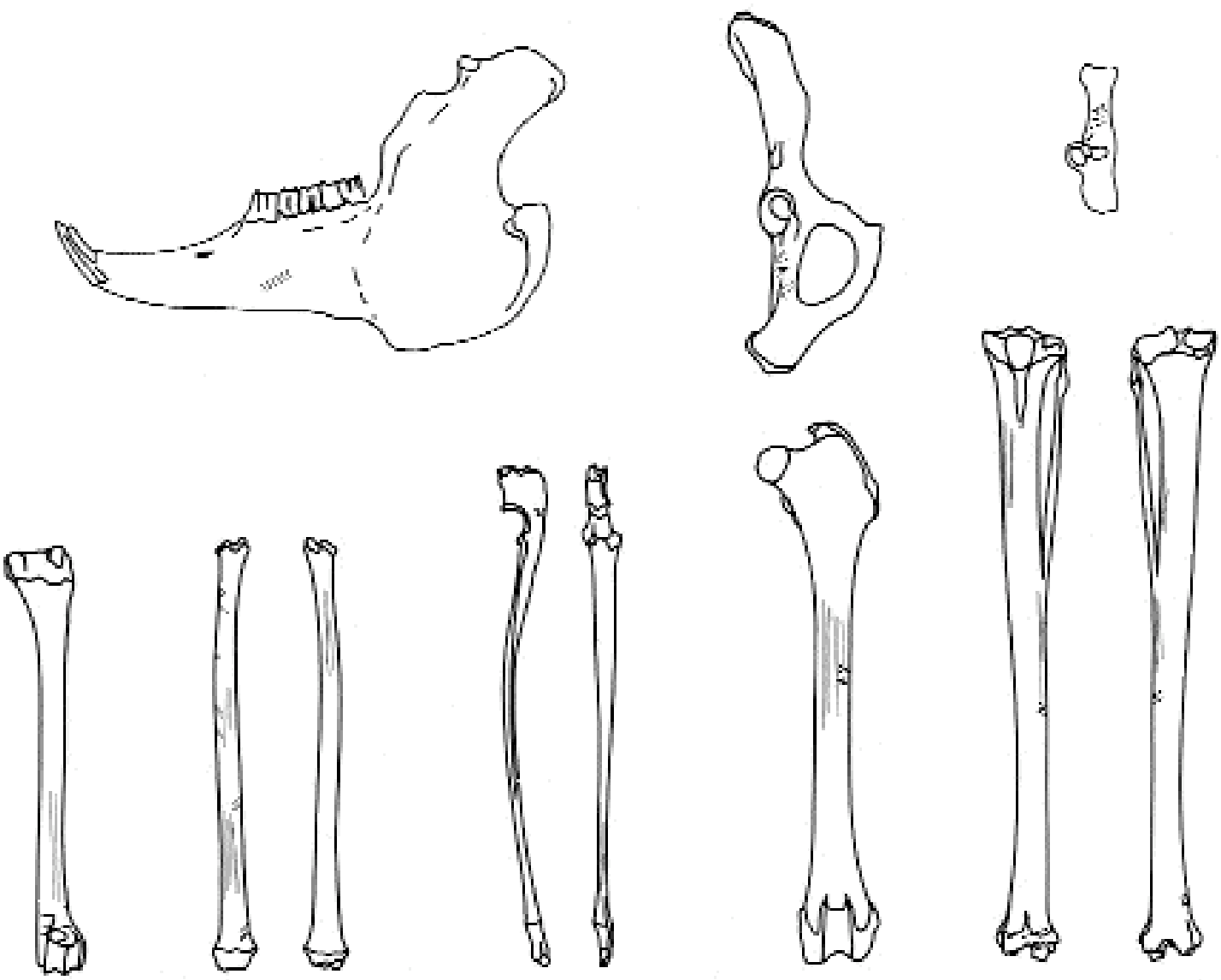

Figure 8 - Emplacement des traces de découpe et de raclage sur les restes de lièvres variables des fouilles Bouvier (couches 26 à 28) et Peyrony (couche inférieure). Dessins modifiés d'après Barone et al. 1973, Morel \& Müller 1997, Tagliacozzo \& Fiore 1998. Seuls les deux diaphyses indéterminées raclées et les deux métatarsiens (fig. 11 et fig. 16) ne sont pas représentés ici.

Figure 8 - Location of cutmarks and scraping marks on Arctic Hare bones (Bouvier's excavations: levels 26 to 28 ; Peyrony's excavations: lower level). Drawings modified after Barone \& al. 1973, Morel \& Müller 1997, Tagliacozzo \& Fiore 1998. Only the two undetermined scraped shafts and the two metatarsals (fig. 11 \& fig. 16) are not figured here. 
Les traces témoignant de la décarnisation se situent au niveau de la diaphyse des os longs et du pelvis. Elles attestent du sectionnement des muscles et/ou de l'enlèvement de la viande crue. Elles sont plutôt courtes et transversales dans le premier cas et plutôt longues, peu profondes et parallèles à l'axe de l'os, dans le second cas. Plus fréquentes que les traces de désarticulation, elles sont visibles sur 4 tibias, 8 radius, un humérus, un fémur et trois coxaux (fig. 10). Leur localisation correspond à ce que l'on a déjà identifié sur les os des lièvres de Champréveyres (Morel et Müller 1997), de Bois Ragot (Cochard 2004), de Gazel (Fontana 2003) et de la grotte Romanelli (Tagliacozzo \& Fiore 1998) avec néanmoins deux différences notables (cf. fig. 8). Aucune trace n'a été détectée sur le col de la scapula alors que la fréquence des traces à cet endroit est très élevée sur les restes issus des quatre sites documentés, et elles sont très fréquentes sur le radius, os qui n'en porte que très rarement dans les gisements évoqués.

Ce sont les traces liées à l'enlèvement de la peau qui sont les plus rares puisque seule la face externe de la branche horizontale d'une mandibule droite porte des traces indéniablement liées au dépouillement (cf. fig. 8) comme on en connaît par exemple à la grotte Romanelli (Tagliacozzo \& Fiore 1998). Dans cette catégorie pourraient éventuellement entrer les multiples traces transversales et profondes figurant sur la face latérale de la diaphyse d'un métatarsien IV (fig. 11). Nous n'avons trouvé aucune équivalence sur les os de Lièvre variable d'autres sites mais il semblerait que de telles traces, existant sur des os de Lapin issus de sites espagnols (Perez Ripoll, comm. pers.), soient interprétées comme des témoins de prélèvement de la fourrure.

D'autres traces ont été identifiées sur 16 os longs (fig.12 et 13), en majorité des radius (5) et des tibias (4) (cf. tabl. 6). II s'agit de traces de raclage qui affectent toute la longueur de la diaphyse, souvent sur plusieurs faces et en double sillon (cf. fig. 8). Les marques de raclage sont associées par certains à la décarnisation des carcasses de lapins (" Grattements allongés de direction longitudinale ", Perez Ripoll 2004, p. 97) et certaines traces sont tout à fait comparables à celles de La Madeleine (Perez Ripoll 2006, p. 254 ; Cochard 2004, p. 235). II semble que l'on ait souhaité ôter toute la chair de ces os mais l'hypothèse d'un décharnement maximal de l'os dans le but de prélever la totalité de la viande ne nous semble pas satisfaisante car les tibias et les radius ne sont pas les os qui en portent le plus, bien au contraire. II pourrait s'agir d'un nettoyage de l'os, destiné à l'élimination du périoste, qui faciliterait ensuite le rainurage mais aucun des fragments en question n'est rainuré. Ils pourraient également avoir été raclés pour être préservés d'une dégradation biologique et avoir été réservés pour une utilisation ultérieure, mais ils auraient été abandonnés sans avoir été transformés. L'hypothèse d'un stockage en vue d'une utilisation différée ne concorde pas non plus avec l'état très fragmentaire de la plupart de ces os.

Les fragments osseux qui portent l'ensemble des traces décrites représentent environ $4 \%$ des restes de Lièvre. Cette proportion est plus faible que celles de la grotte
Romanelli (6,3 \%, Tagliacozzo \& Fiore 1998), de Bois Ragot (environ $8 \%$, Cochard 2004), de Champréveyres (environ $13 \%$, Morel et Müller 1997) et de Robin Hood Cave (22 \%, Charles \& Jacobi 1994) alors qu'elle est forte par rapport à celle de Gazel (0,5\%, Fontana 2003) et d'autres sites où aucun reste ne porte de traces comme Belvis (Fontana 1998), Les Eglises (Delpech et Le Gall 1983) ou encore Combe-Saunière (Castel 1999). Ces traces confirment le prélèvement de la fourrure, suggéré par l'absence des vertèbres caudales et des patellas, mais sans préciser son caractère systématique ou anecdotique. Elles suggèrent également que certaines parties ont été désarticulées et décharnées au silex sans que l'on puisse évaluer la fréquence de ces deux types d'action. Enfin, aucune trace d'altération thermique ne permet d'argumenter l'hypothèse de la cuisson de la viande par rôtissage.

Six os portent les stigmates de deux procédés techniques, le rainurage et le sciage au silex. Ils renvoient à la production d'un outillage et d'une parure de petites dimensions (tabl. 12). Ainsi, quatre os (deux tibias, un radius et un fémur) portent, sur leur face antérieure, les traces d'un débitage par rainurage longitudinal multiple, destiné à l'extraction de baguettes rectilignes. Elles ont pu être transformées par la suite en aiguilles à chas, comme dans d'autres gisements magdaléniens (fig.14). L'épaisseur de tissu compact de ces matrices peut être rapprochée de celle des aiguilles de La Madeleine (fig. 15). La faible épaisseur de compacta des os longs de Lièvre permet une extraction rapide de volumes dont le calibre est proche des objets finis, ce qui diminue d'autant l'étape du façonnage. Par conséquent, en dehors des matrices, ce travail engendre peu de déchets de fabrication et peut-être même parfois aucun déchet de résection. II est difficile de faire le lien entre le nombre total d'aiguilles à chas et la production estimée des seules matrices. Toutefois, on peut fortement présumer d'une fabrication partielle in situ des premières à partir des secondes. Un métatarsien III de la collection Bouvier (couche 28) présente les traces d'un sectionnement transversal de la diaphyse au silex (fig. 16). Le court cylindre produit a pu être transformé en tube ou servir à la confection d'une imitation de test de dentalium, comme sur le site de Petersfels en Allemagne (Albrecht et al. 1983). Enfin, une diaphyse de tibia droit (avec l'extrémité proximale, cf. tabl. 6) issue de la série Peyrony présente une " perforation " particulière. Cette pièce, par ailleurs raclée sur l'autre face, possède un trou ovale localisé sur la crête tibiale (fig. 17). Les parois de cet orifice sont altérées par un effritement de la matière et elles sont d'une patine plus claire que celle du corps de l'os. L'examen à la loupe binoculaire n'a pas révélé de traces de façonnage telles que des stries de rotation ou des lunules de pression. D'ailleurs, aucun objet fonctionnellement efficace réalisé sur os de Lièvre variable et muni d'un trou de ce calibre n'a jusqu'à présent été identifié dans l'outillage ou la paru re paléolithique. II ne peut s'agir non plus d'une marque produite par un carnivore. En revanche, l'action d'une racine ou la dissolution chimique ponctuelle de l'os pourrait rendre compte de cette modification. Cette pièce peut être rapprochée d'une 

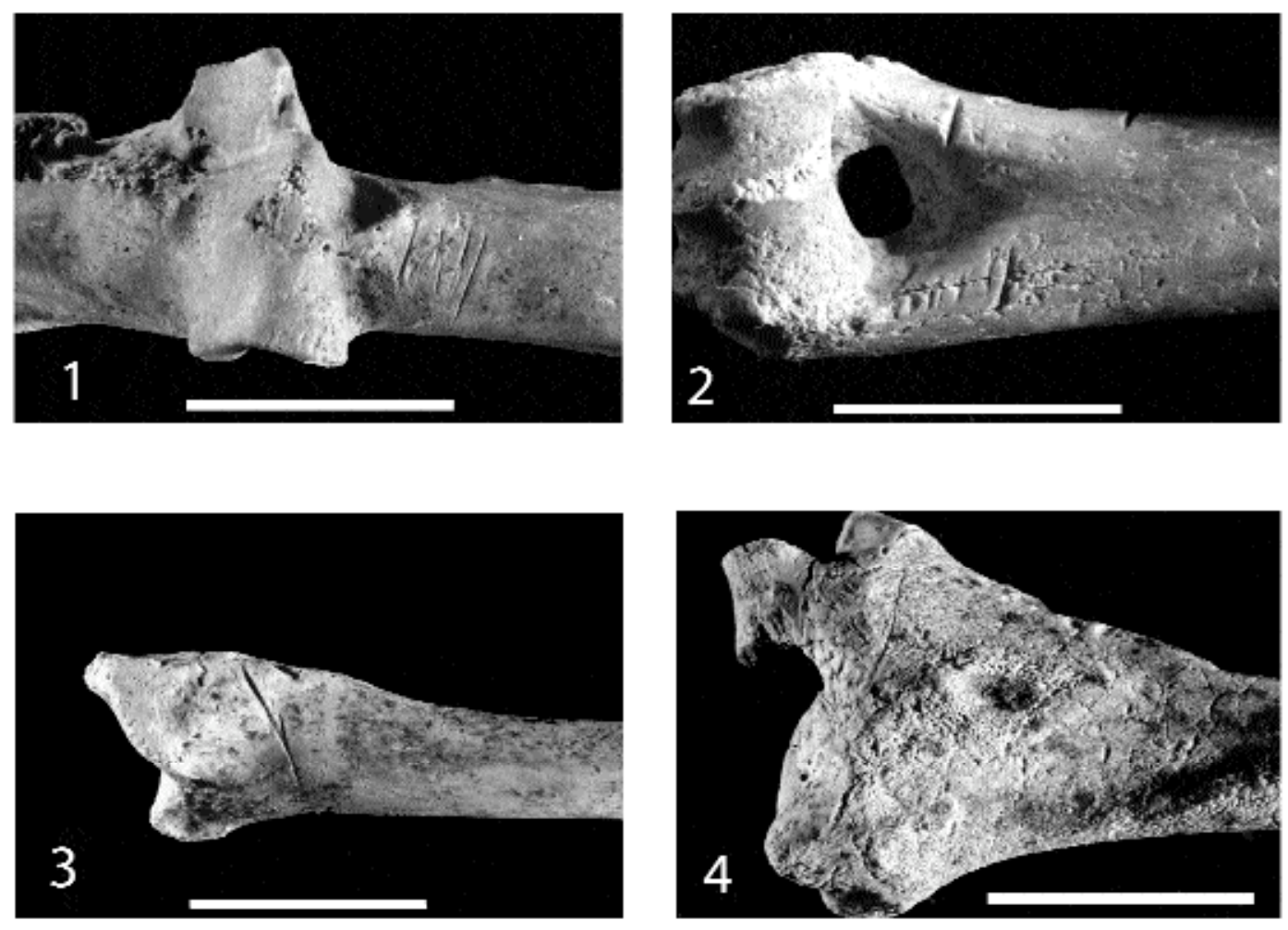

Figure 9 - Lièvre variable : Traces de désarticulation. 1- Calcanéum droit (collection Peyrony, couche inférieure) ; 2- Humérus droit (collection Peyrony, couche inférieure) ; 3- Radius droit (collection Peyrony, couche inférieure) ; 4- Tibia droit (collection Peyrony, couche inférieure). Clichés Ph. Jugie (MNP).

Figure 9 - Arctic Hare: Dismembering marks. 1- Right calcaneus (Peyrony's collection, lower level) ; 2- Right humerus (Peyrony's collection, lower level) ; 3- Right radius (Peyrony's collection, lower level) ; 4- Right tibia (Peyrony's collection, lower level). Photos: Ph. Jugie (MNP).

côte complète de Lièvre variable du Magdalénien du Kesslerloch (Suisse) présentant une perforation allongée analogue. De patine également plus claire que le reste de la surface osseuse, cet orifice est considéré par J. Heierli (1907, p. 176-177, pl. XIX, $n^{\circ}$ 3) comme le résultat d'un percement anthropique (cf fig. 17). Même si les os de Lièvre de la Madeleine ont été avant tout utilisés pour produire des aiguilles à chas, cette exploitation apparaît très réduite, comme c'est le cas dans les autres sites où elle est attestée (tabl. 13). Elle peut même être considérée comme anecdotique puisque les aiguilles ont été essentiellement réalisées à partir des os de Renne et de Cheval (Capitan et Peyrony 1928). De même, les os de Lièvre ne semblent pas avoir fait l'objet d'une sélection pour la fabrication de la parure alors que cette catégorie est riche de plusieurs autres taxons et matériaux (dents, coquille, pierre) (Capitan et Peyrony op. cit. ; Taborin 1993).

L'ensemble des traces identifiées sur les os de Lièvre variable de La Madeleine atteste de la réalisation de plusieurs types d'opération menées sur les carcasses, depuis l'écorchement jusqu'à la décarnisation via la désarticulation, et le prélèvement de matière osseuse. C'est la part relative de ces différentes actions et donc la nature de l'exploitation qui sont plus délicates à appréhender. L'étude de la fracturation osseuse peut-elle contribuer à résoudre cette question?

\section{3 - La fracturation}

Identifier la nature et l'intensité de la fracturation des restes de Lièvre a constitué l'exercice le plus délicat de cette étude, qu'il s'agisse de la série Peyrony dont on n'a collecté que les restes quasi entiers ou de la série Bouvier dont les effectifs n'excèdent pas 248 pour les couches 28 et 27 (cf. tabl. 3). Néanmoins, la morphologie des fractures et la représentation des différentes portions osseuses livrent certaines informations significatives.

Dans la collection Peyrony, les os longs sont presque exclusivement des diaphyses dotées de leur extrémité distale, plus rarement proximale et seuls les métapodes sont entiers (cf. tabl. 7). Ce sont uniquement des cylindres entiers dont la taille varie, depuis une diaphyse quasi entière jusqu'au quart de la diaphyse, la majorité étant d'un tiers de la longueur (fig. 18.1 et 18.2). Les restes de la collection Bouvier sont plus divers : extrémités, fragments de dia- 

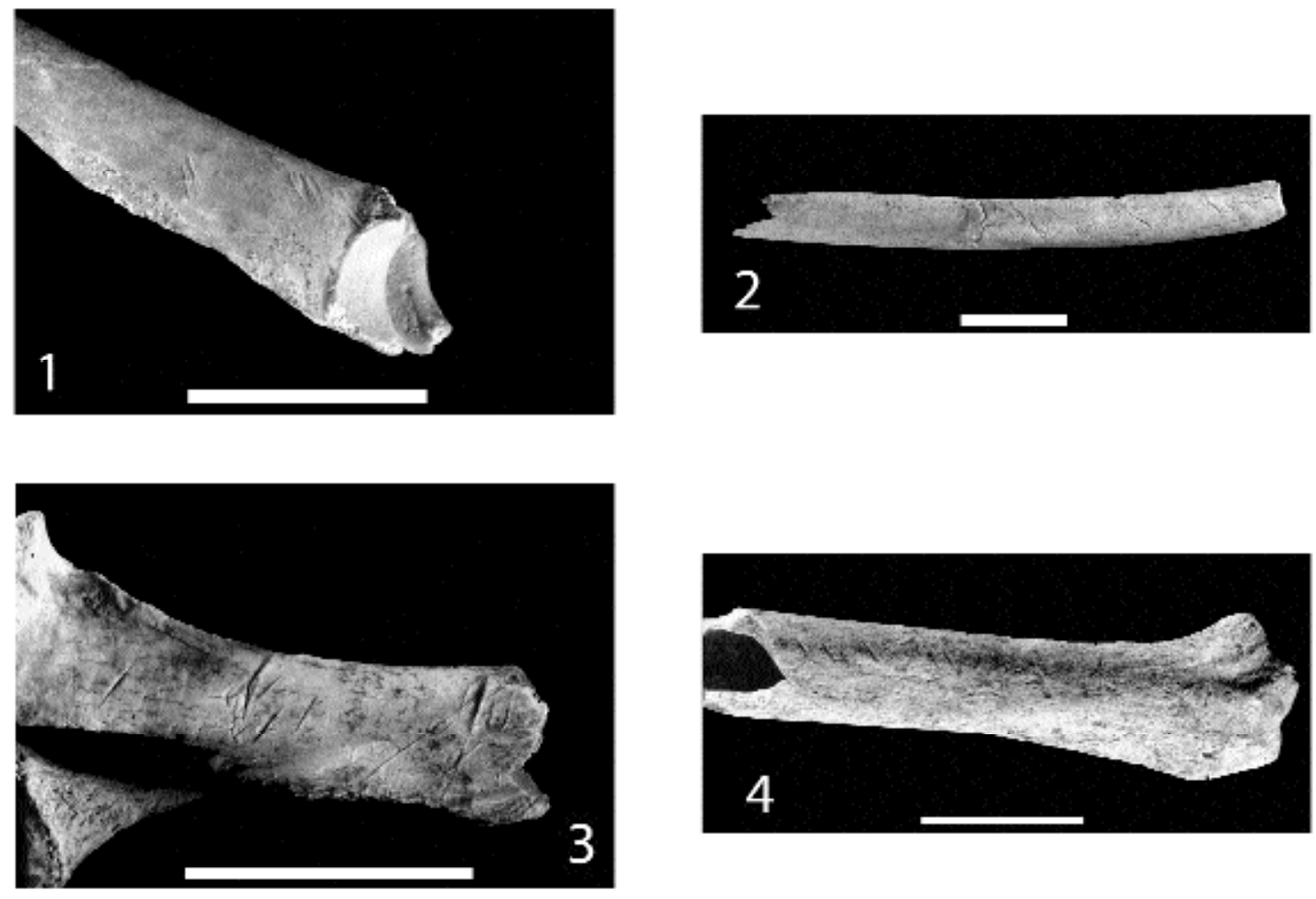

Figure 10 - Lièvre variable: Traces de décarnisation. 1- Radius gauche (collection Bouvier, couche 26, K 17) ; 2- Radius gauche (collection Bouvier, couche 26 F, L 17) ; 3- Coxal droit (collection Peyrony, couche inférieure) ; 4- Radius droit (collection Peyrony, couche inférieure). Clichés Ph. Jugie (MNP).

Figure 10 - Arctic Hare: Butchering marks. 1- Left radius (Bouvier's collection, level 26, $K$ 17) ; 2- Left radius (Bouvier's collection, level 26 F, L 17) ; 3- Right pelvis (Peyrony's collection, lower level) ; 4- Right radius (Peyrony's collection, lower level). Photos: Ph. Jugie (MNP).

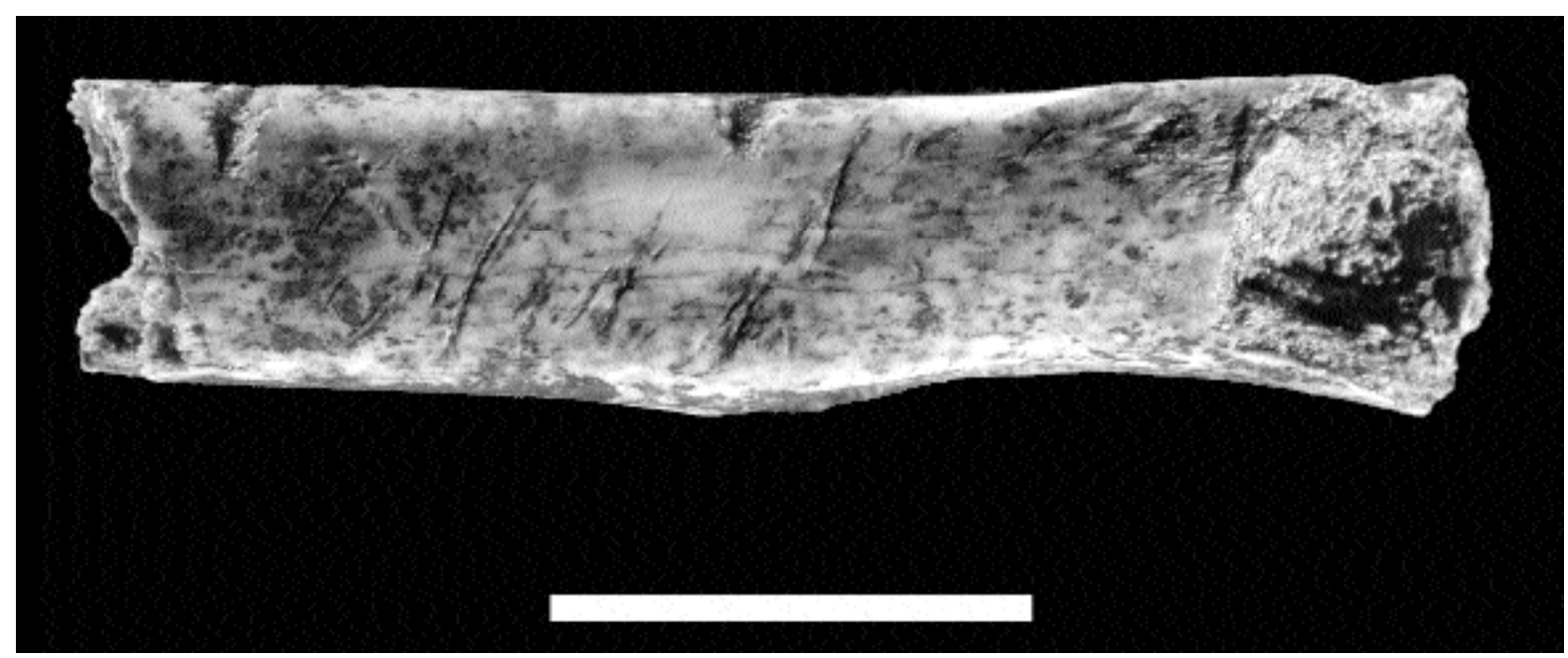

Figure 11 - Métatarsien IV droit de Lièvre variable (collection Bouvier, couche 27, H 18) Cliché Ph. Jugie (MNP).

Figure 11 - Arctic Hare right metatarsal IV (Bouvier's collection, level 27, H 18). Photo Ph. Jugie (MNP). 

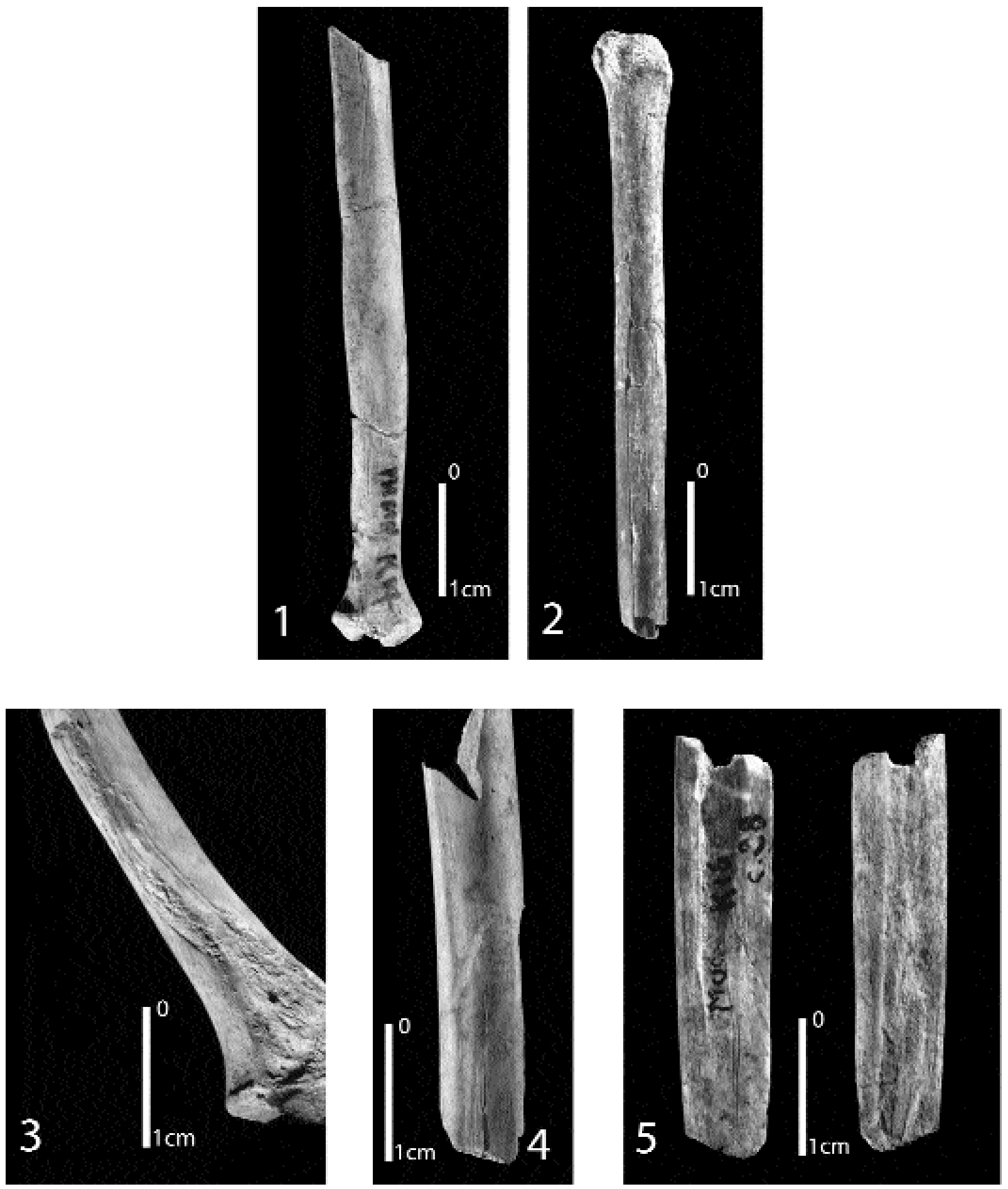

Figure 12 - Os raclés de Lièvre variable. Il s'agit d'un raclage léger dont la finalité technique est équivoque. 1- Radius gauche (collection Bouvier, couche 26, K17), 2- Radius droit (collection Bouvier, couche 28, 116); 3- Radius gauche (collection Peyrony, couche inférieure) ; 4- Humérus (collection Bouvier, couche 26 F, K17) ; 5- Ulna (collection Bouvier, couche 28, J17). Clichés Ph. Jugie (MNP).

Figure 12 - Arctic Hare scraped bones. It is a slight scraping whose technical aim is unknown. 1- Left radius (Bouvier's collection, level 26, K17), 2- Right radius (Bouvier's collection, level 28, 116) ; 3- Left radius (Peyrony's collection, lower level) ; 4Humerus (Bouvier's collection, level 26 F, K17) ; 5- Ulna (Bouvier's collection, level 28, J17). Photos: Ph. Jugie (MNP). 

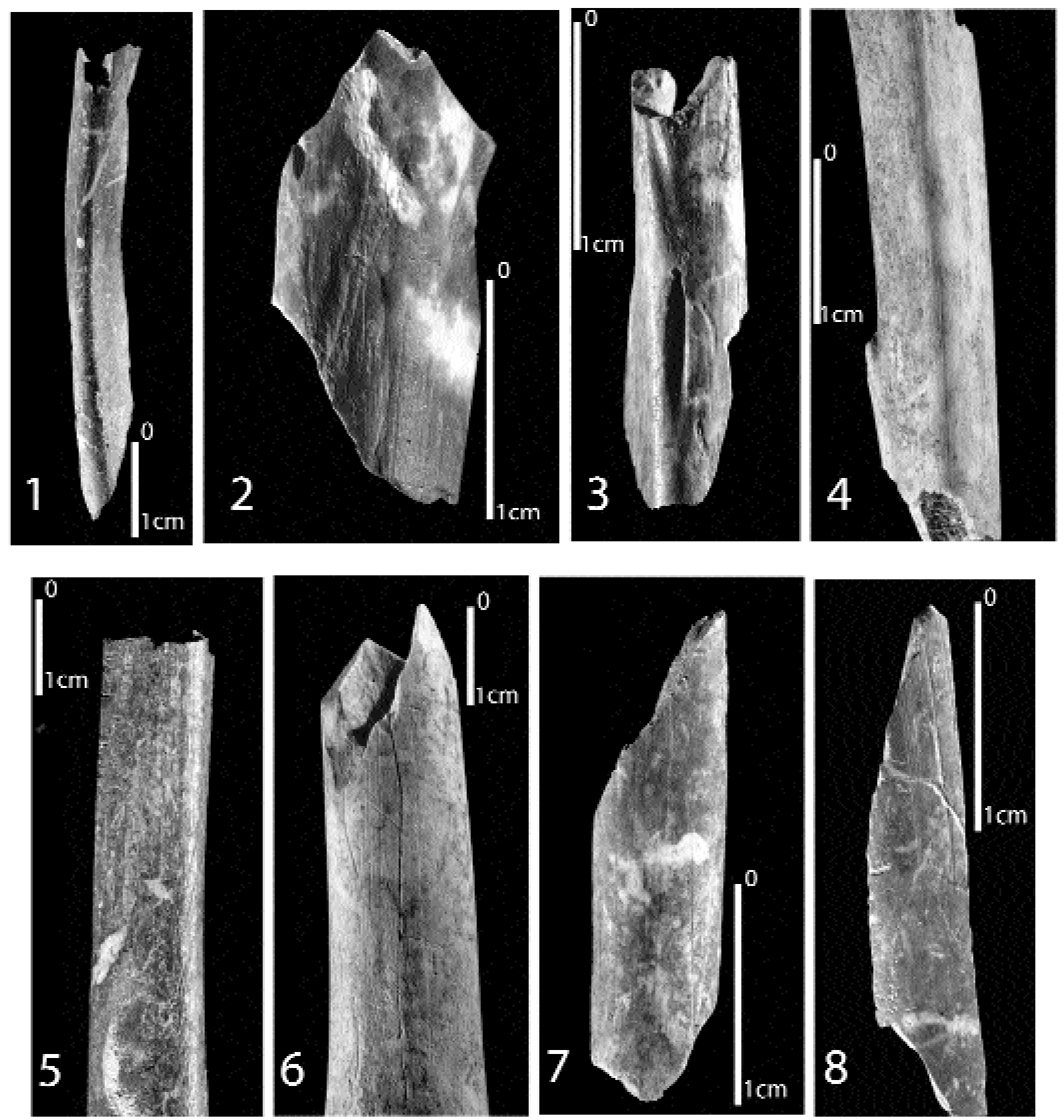

Figure 13 - Os raclés de Lièvre variable. Il s'agit d'un raclage léger dont la finalité technique est équivoque. 1- Fémur droit (collection Bouvier, sondage 29) ; 2- Fémur droit (collection Bouvier, couche 27, M17) ; 3- Tibia droit (collection Bouvier, couche 28, J18) ; 4- Tibia (collection Bouvier, couche 26, L27) ; 5- Tibia gauche (collection Bouvier, couche 27, K16) ; 6- Fémur droit (collection Peyrony, couche inférieure) ; 7- Diaphyse indéterminée (collection Bouvier, couche 28, 118) ; 8- Diaphyse indéterminée (collection Bouvier, couche 27, K18). Clichés Ph. Jugie (MNP).

Figure 13 - Arctic Hare scraped bones. It is a slight scraping whose technical aim is unknown. 1- Right femur (Bouvier's collection, drilling 29) ; 2- Right femur (Bouvier's collection, level 27, M17) ; 3- Right tibia (Bouvier's collection, level 28, J18) ; 4- Tibia (Bouvier's collection, level 26, L27) ; 5- Left tibia (Bouvier's collection, level 27, K16) ; 6- Right femur (Peyrony's collection, lower level) ; 7- Undetermined shaft (Bouvier's collection, level 28, 118) ; 8- Undetermined shaft (Bouvier's collection, level 27, K18). Photos: Ph. Jugie (MNP). 


\begin{tabular}{|c|c|c|c|c|}
\hline Série - Couchẹ & Anatomic & Stigmale & Calćgoric & Production \\
\hline Coll. Bouvier C. 27 (J18) & Fćmur droit & rainurage & matrice & aiguilles \\
\hline (oll. Bouvier (C. 27 (M19) & Tibia gauche & rainurage & malrice & aisuilles \\
\hline Coll. Bouvicr C. 28 (H.J18) & Diaphysc indćt. & rainurage & bagucttc & aiguilles \\
\hline Coll, Bouvier (C. 28 (J18) & Mélalarsięn III & sciage au trail de silex & matrice & lube \\
\hline \multirow{2}{*}{$\begin{array}{l}\text { Coll. P’cyrony } \\
\text { C. inlériecure }\end{array}$} & Radius droit & rainurage & matrice & aiguilles \\
\hline & Tibią droit & rạinuruge & malrice & aisuilles \\
\hline
\end{tabular}

Tableau 12 - Détail des restes osseux travaillés de Lièvre.

Table 12 - Detail of Arctic Hare worked bones.
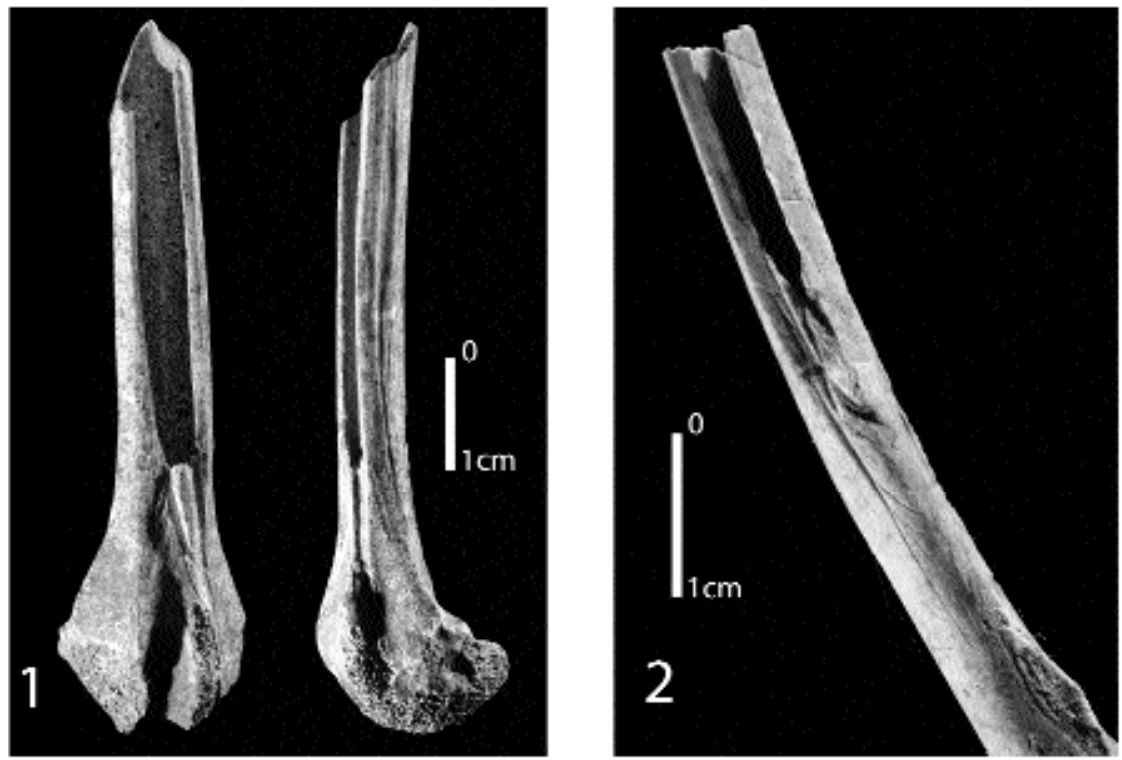

Figure 14 - Os de Lièvre variable débités par rainurage afin d'extraire des baguettes pour la production d'aiguilles à chas. 1 Fémur droit (collection Bouvier, couche 27, J18), 2Radius droit (collection Peyrony, couche inférieure) ; 3- Tibia droit (collection Peyrony, couche inférieure) ; 4- Tibia gauche (collection Bouvier, couche 27, M19) ; 5Diaphyse indéterminée (collection Bouvier, couche 28, HJ18). Clichés Ph. Jugie (MNP).

Figure 14 - Artic Hare needles matrices. 1- Right femur (Bouvier's collection, level 27, J18), 2- Right radius (Peyrony's collection, lower level) ; 3- Right tibia (Peyrony's collection, lower level) ; 4- Left tibia (Bouvier's collection, level 27, M19) ; 5- Undetermined shaft (Bouvier's
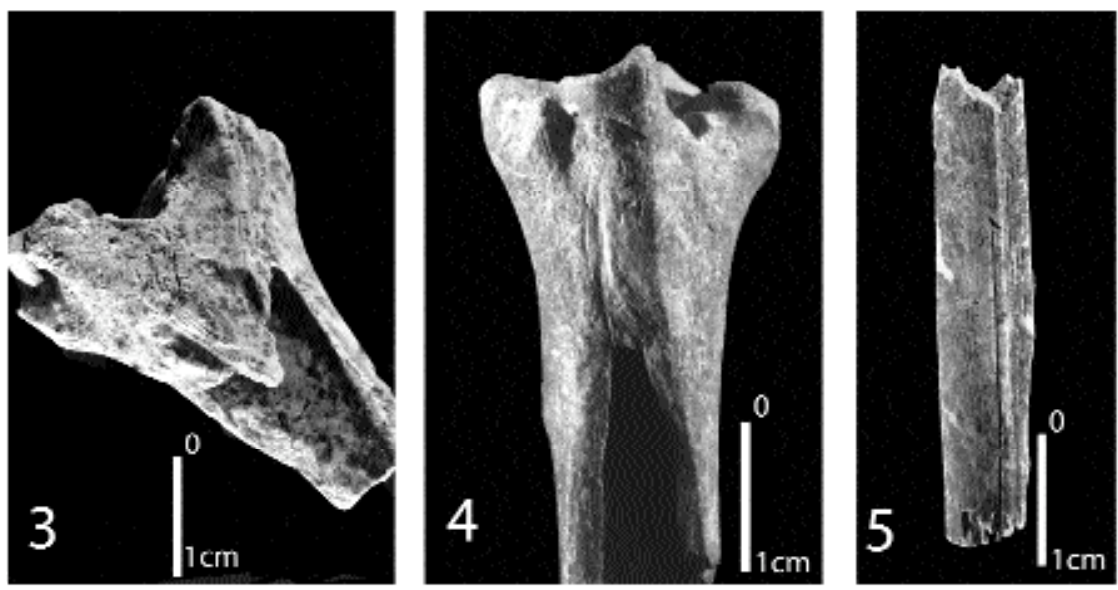
collection, level 28, HJ18). Photos: Ph. Jugie (MNP). 


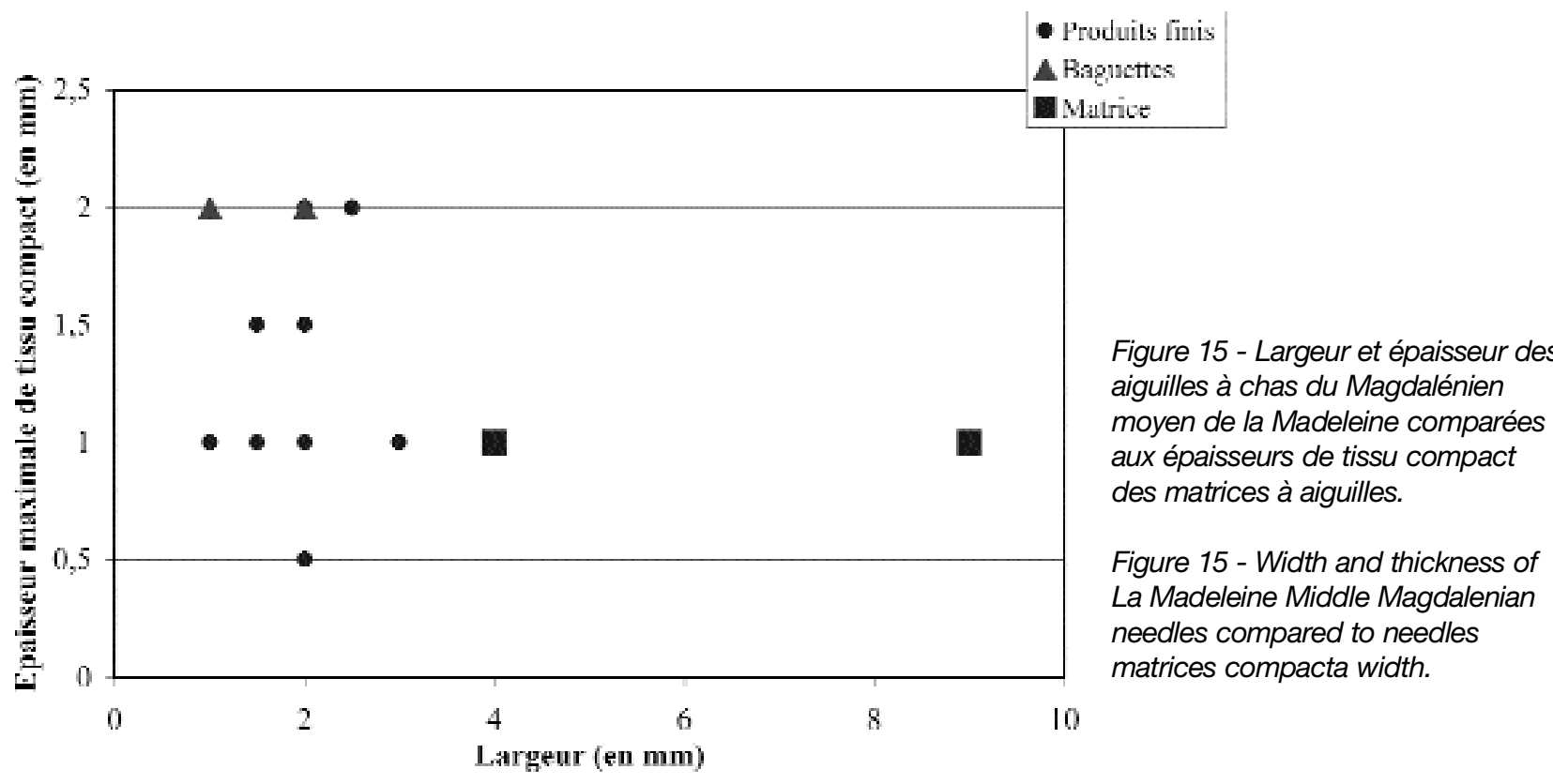

physe (cylindriques ou non) avec ou sans extrémité (fig. 18.3 à 18.6). La taille moyenne des restes d'os longs est très inférieure à celle des restes de la collection Peyrony mais les os longs complets sont tout aussi rares. La nature de ces deux séries, collectée au profit des grands fragments pour l'une et constituée d'effectifs très limités pour l'autre, rend inutile toute tentative de préciser l'intensité de la fracturation (par exemple par le calcul NRD/NMPS) une fois que l'on a rappelé qu'aucun os long n'était complet, excepté quatre métapodes de la série Bouvier et les 227 des 342 métapodes de la série Peyrony. II s'agit donc d'une fracturation intense des os longs qui ne visait pas systématiquement les métapodes et qui peut être en partie d'origine sédimentaire. Examinons donc de plus près les types de fracture afin d'identifier leur origine respective.

La grande majorité des fractures des humérus, des fémurs et des tibias ont une morphologie identique, hélicoïdale ou en spirale, aux bords plutôt lisses et obliques (fig. 18.2, $18.3,18.5)$, et ce, quelle que soit leur taille ou leur nature (diaphyse ou épiphyse). Ceci résulte, d'après les critères d'identification admis (Morlan 1984 ; Johnson 1985), d'une fracturation des os à l'état frais. Si une partie des diaphyses de radius est également fracturée de cette façon, d'autres (fig. 18.1 et 18.6) présentent des fractures transversales au bord plus ou moins dentelé et à angle droit, plutôt caractéristiques d'une fracturation d'os sec qui ressemblent à celles décrites à Bois Ragot (Cochard 2004). II est donc possible qu'une partie des radius (et des ulnas, rarissimes dans les deux séries) n'ait pas été fracturée lors du traitement des carcasses. D'après les référentiels actuels et archéologiques disponibles pour les lagomorphes (notamment Perez Ripoll 1993, 2004 et 2005/2006), la distinction entre les différents modes de fracturation (percussion, flexion et pression dentaire humaine) semble claire. Si la fracturation par pression dentaire est la plus évidente à diagnostiquer, nous n'avons identifié, sur les restes des lièvres de La Madeleine, aucu- ne marque qui soit comparable à celles qui sont décrites pour les restes de lapins du site gravettien espagnol de les Cendres (Perez Ripoll 2004 et 2005/2006). La distinction fracture par percussion/fracture par flexion nous a semblé, quant à elle, nettement moins évidente : la première est censée laisser des encoches caractéristiques et des bords irréguliers ; la seconde, une cassure typique plutôt en spirale pour certains (Cochard 2004), plutôt " nette, transversale " (Perez Ripoll 2004, p. 198) pour d'autres. Si les fractures, déjà décrites, des bords de diaphyses nous semblent plutôt caractéristiques d'une fracturation par flexion (cf. fig. 18), certaines pièces traduisent néanmoins l'utilisation de la percussion.

L'enjeu de la caractérisation de la fracturation est en partie lié à la question de l'extraction de la moelle et de la graisse osseuse. De ce point de vue, les restes de Lièvre de La Madeleine livrent, semble-t-il, deux types d'information. D'une part, les cylindres et les extrémités seules, qui attesteraient d'une récupération de la moelle (Hockett 1991 et 1995 ; Morel et Müller 1997 ; Perez Ripoll 1993 ; Schmitt 1990) sont rares dans les deux collections (cf. supra). Les diaphyses d'os longs dotées d'une des deux extrémités sont majoritaires et témoignent d'un autre type de cassure, par flexion, également documenté dans d'autres sites (Hockett \& Bicho 2000 ; Perez Ripoll 2002). La seconde information livrée par l'observation de ces diaphyses est relative à leur longueur qui est très variable, et ce, dans les deux collections : certains os longs ont seulement été fracturés en deux, la cassure s'effectuant de façon variable au milieu de la diaphyse ou plus à proximité d'une des deux extrémités, alors que d'autres ont été nettement plus fracturés. Par conséquent, s'il est fort possible que l'extraction de la moelle soit à l'origine de la fracturation de certains os longs, le diagnostic nous semble plus délicat pour tous les grands fragments de diaphyses dotées d'une de leurs deux extrémités (radius exclus, si on considère l'origine de leur fracturation, en partie post-dépositionnelle). Ce mode de 


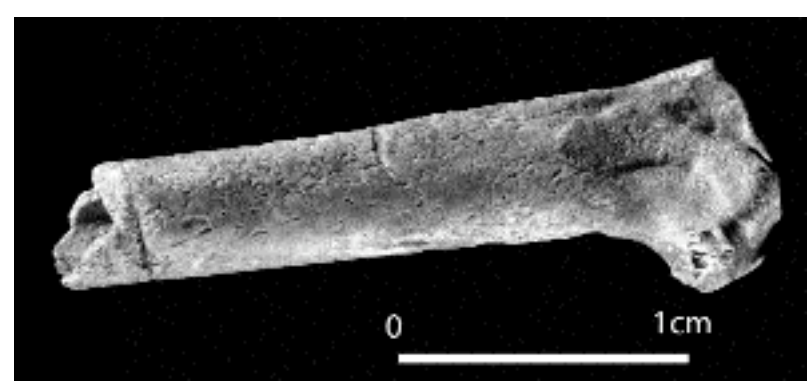

Figure 16 - Métatarsien III de Lièvre variable sectionné au milieu de la diaphyse au trait de silex. La partie manquante a pu servir à la réalisation d'un tube ou d'une imitation d'un test de Dentalium (collection Bouvier, couche 28, J18). Cliché : Ph. Jugie (MNP).

Figure 16 - Arctic Hare sewed metatarsal III. The missing part could be used for a tube manufacture or a Dentalium shell copy (Bouvier's collection, level 28, J18). Photos: Ph. Jugie (MNP).
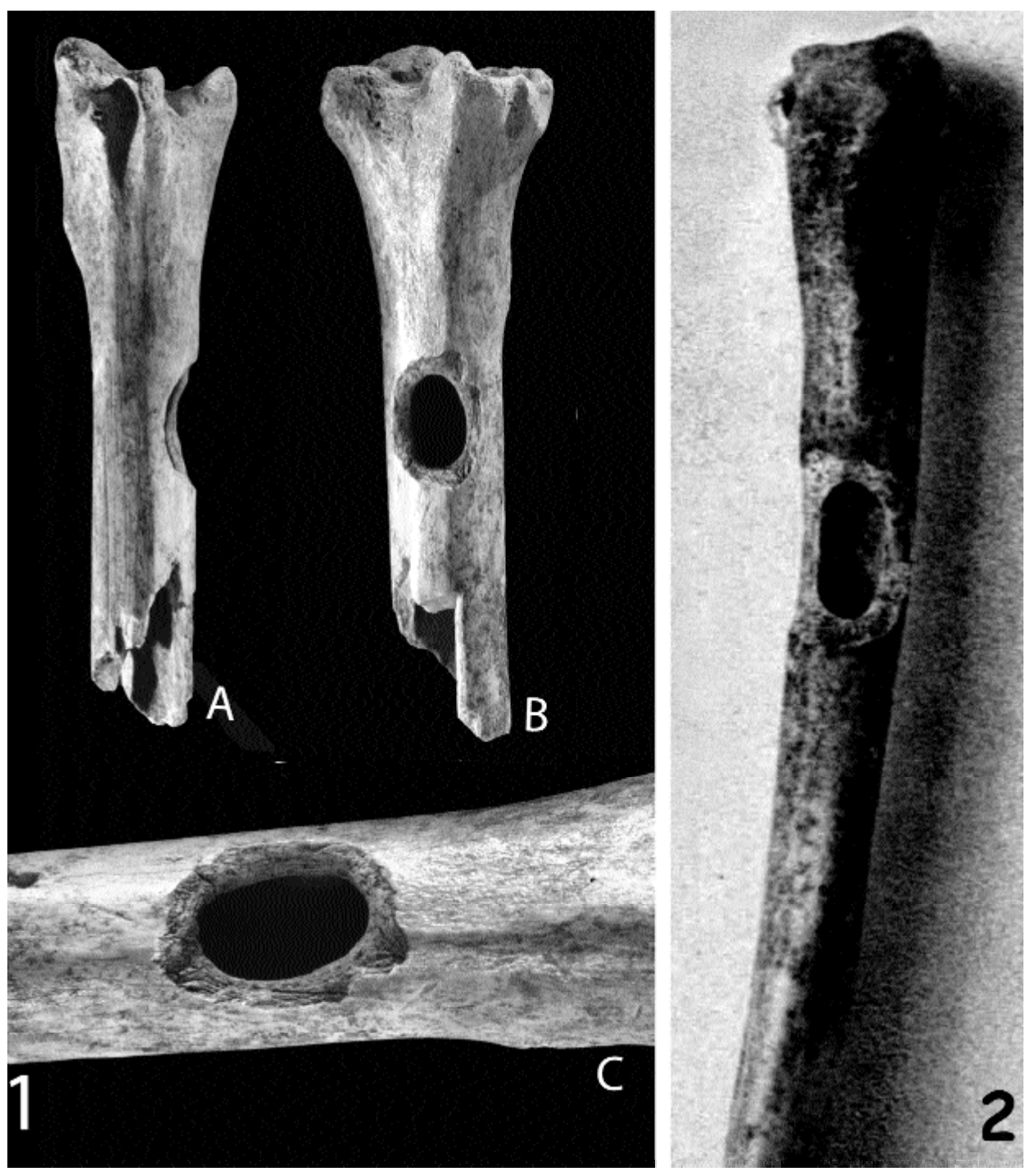

Figure 17 - 1- Tibia droit de Lièvre variable avec perforation sur la face antérieure (collection Peyrony, couche inférieure). L'origine de la perforation n'est pas établie. Cliché Ph. Jugie (MNP) ; 2- Pour comparaison, côte de Lièvre de Kesslerloch (Suisse) présentant le même type de perforation (d'après Heierli 1907, modifié).

Figure 17 - 1- Arctic Hare right tibia with a perforation on the dorsal side (Peyrony's collection, lower level). The origin of the perforation is still unknown. Photo Ph. Jugie (MNP) ; 2- Hare's rib of Kesslerloch (Switzerland) wearing a similar type of perforation (after Heierli 1907, modified). 


\begin{tabular}{|c|c|c|c|c|}
\hline SITES & $\mathrm{MN} / \mathrm{BG}$ & Tube & Poinçon & Parure \\
\hline \multicolumn{5}{|c|}{ Bois Ragot (niveau 5) } \\
\hline Radius & 7 & & & \\
\hline Ulna & & & 1 & \\
\hline Fémur & 1 & & & \\
\hline Tibia & 29 & & & \\
\hline Indétcrminé & 6 & & & 2 \\
\hline \multicolumn{5}{|c|}{ Combe Saunière (C: 4) } \\
\hline Tibia & & & 1 & \\
\hline \multicolumn{5}{|c|}{ La Madeleine (M IV) } \\
\hline Radius & 1 & & & \\
\hline Tibia & 2 & & & \\
\hline Fémur & 1 & & & \\
\hline \multicolumn{5}{|l|}{ La Vache } \\
\hline Fémur & & 1 & & \\
\hline \multicolumn{5}{|c|}{ (iazel (couche 7) } \\
\hline Incisive & & & & 1 \\
\hline \multicolumn{5}{|c|}{ P'etersfels (tous niv.) } \\
\hline Tibia & non précisć & & & \\
\hline Métapode & & & & 15 \\
\hline \multicolumn{5}{|c|}{ Champréveyres } \\
\hline Tíbia & 6 & & & \\
\hline Indéterminé & $5 \pm 50^{\circ}$ & & & \\
\hline \multicolumn{5}{|l|}{ Monruz. } \\
\hline Humćrus & 3 & & & \\
\hline Tibia & 5 & & & \\
\hline Indélerminé & $2 \pm 39^{\neq}$ & & & \\
\hline \multicolumn{5}{|c|}{ Schweizersbild } \\
\hline Tibia & non précisć & & & \\
\hline \multicolumn{5}{|l|}{ Kesslerloch } \\
\hline Tibia & non précisć & & & \\
\hline
\end{tabular}

Tableau 13 - Restes osseux de Lièvre travaillés dans les sites du Magdalénien et du Solutréen d'Europe occidentale (* : Aves/Lepus ; MA : matrice à aiguilles, $B G$ : baguette).

Références : Albrecht et al. 1983, Averbouh 2003, Bullinger et al. 2006, Chauvière \& Castel 2004, Heierli 1907, Hescheler 1907, Höneisen \& Peyer 1994, Leesch 1997, Morel \& Müller 1997, Nuëch 1896, Sacchi 2003.

Table 13 - Hare worked bones in western european Magdalenian and Solutrean sites (*: Aves/Lepus ; MA: needles matrice, BG: baguette).

Literature cited: Albrecht \& al. 1983, Averbouh 2003, Bullinger \& al. 2006, Chauvière \& Castel 2004, Heierli 1907, Hescheler 1907, Höneisen \& Peyer 1994, Leesch 1997, Morel \& Müller 1997, Nuëch 1896, Sacchi 2003.

fracturation traduit-il une autre technique de récupération de la moelle (le " bouillon ") ou bien un autre objectif ?

\section{4 - Une exploitation ciblée ?}

A l'issue de l'analyse des données liées aux modalités d'exploitation des lièvres, plusieurs faits semblent établis. Ainsi, plusieurs produits ont été indéniablement prélevés sur les carcasses : la fourrure, la chair crue, des os et de la moelle osseuse. On peut aussi supposer l'utilisation de la fourrure, la consommation de la moelle et démontrer l'utilisation de l'os. En revanche, on ignore si la viande c rue a été consommée et si de la viande a été cuite dans le même but. Le raclage, dont témoignent 18 diaphyses, atteste d'une volonté d'ôter toute la matière carnée, ce qui n'implique pas de facto la consommation de la chair. La part respective de ces prélèvements et le ou les objectif(s) de l'exploitation sont-ils accessibles à partir de ces résultats ? Les données relatives aux traces et à la fragmentation indiquent que chaque produit fut prélevé pour une petite part : une seule trace sur une mandibule attes- te du prélèvement de la fourrure, de rares traces témoignent du prélèvement de la viande crue et six restes ont été utilisés à des fins techniques. Seul le prélèvement de la moelle pourrait avoir été plus systématique. Néanmoins, nous savons que la récupération de la moelle, si elle s'effectue par fracturation des os, est plus aisément identifiable que la consommation de viande crue ou le prélèvement de la peau. C'est pourquoi nous précisions, au début de ce chapitre, que ces témoignages doivent être analysés ensemble, au regard de la représentation des parties squelettiques et, surtout, replacés dans le contexte de l'exploitation des autres gibiers. En effet, en examinant les produits prélevés sur les carcasses de Lièvre et sur celles des autres gibiers, on constate que le Renne (et dans une moindre mesure le Cheval) fut chassé dans le but d'acquérir des produits alimentaires (viande et moelle) et des matériaux divers (appendices frontaux, os, peau, tendons...). A La Madeleine, les matières osseuse et carnée furent donc sans aucun doute majoritairement fournies par le Renne. Si on ajoute à cela le fait que la fourrure du Lièvre est un des seuls produits qui ne 

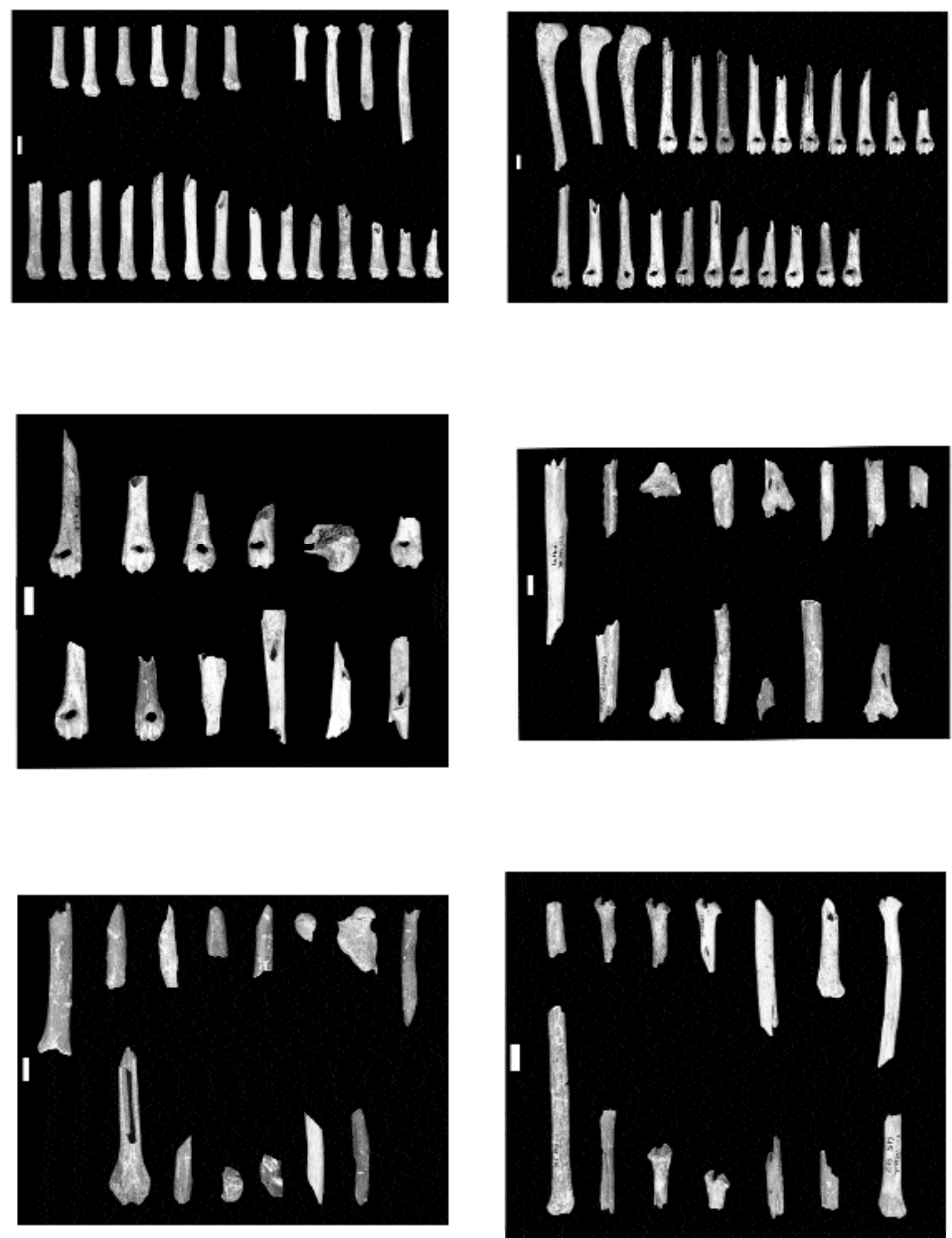

Figure 18 - Exemples de la fragmentation des os longs de Lièvre variable dans les collections Peyrony (1-Radius ; 2- Humérus) et Bouvier (3- Humérus ; 4- Tibias ; 5- Fémurs ; 6- Radius). Clichés Ph. Jugie (MNP).

Figure 18 - Examples of Artic Hare long bones fragmentation in the Peyrony's (1- Radius ; 2- Humerus) and Bouvier's (3- Humerus ; 4- Tibias ; 5- Femurs ; 6- Radius) collections. Photos: Ph. Jugie (MNP). 
trouve pas d'équivalent chez le Renne ni chez aucun autre animal, on peut se demander si l'exploitation du Lièvre n'était pas centrée sur la récupération de la fourrure, comme à la grotte Gazel où elle fut très probablement le seul produit recherché. Le prélèvement de diaphyses pour la confection d'aiguilles fut anecdotique alors que la récupération de la moelle a pu être pu systématique mais tout autant opportuniste. II est d'ailleurs possible que le seul but de la décarnisation ait été d'accéder à la moelle. II n'est pas possible de rejeter l'hypothèse de la consommation de viande cuite, qui n'aurait laissée aucune trace (même de brûlure). Mais il est plus di fficile de considérer que l'objectif premier de l'acquisition du Lièvre fut la consommation de viande, sur un site où rennes et chevaux ont été désossés en quantité. L'hypothèse d'une exploitation ciblée de la fourrure nous semble donc être celle qui prend en compte l'ensemble des données, notamment le choix des individus adultes.

\section{CONCLUSIONS}

Au terme de cette étude, il nous semble que les caractéristiques de l'exploitation du Lièvre variable à La Madeleine contribuent à la connaissance du statut du Lièvre au Magdalénien moyen. Néanmoins, si ce gisement est l'un des trois sites témoignant d'une capture importante de lièvres variables, ce n'est que dans une moindre mesure. En effet, la quantité de restes exhumés est faible (1 035 restes) comparée à celle des restes de Lièvre de Gazel (8 000 restes) et Kesslerloch (15 000 restes) et elle ne s'explique pas uniquement par le caractère sélectif de la collecte des fouilles anciennes. Pourtant, sa représentation à La Madeleine (moins d'un quart des restes fauniques) ne renvoie pas pour autant le Lièvre variable au statut de gibier complémentaire dont l'acquisition aurait été plus ou moins opportuniste. En effet, quand on connaît la rareté des témoins qui attestent du prélèvement de la fourrure d'autres animaux (par exemple l'Isatis) on soupçonne que le Lièvre variable fut, au moins entre 15000 et $14000 \mathrm{BP}$, considéré avant tout comme un animal à fournure, voire L'animal à fourrure. Cette idée n'est pas nouvelle (voir Lartet et Christy 1864-1875) et c'est surtout le doute quant à l'importance de la consommation de sa viande qui a attiré l'attention sur l'exploitation de cet animal (Müller 2004 ; Fontana 2003 et 2004). On doit maintenant se demander si cette hypothèse de prélèvement préférentiel, voire exclusif, de la fourrure de Lièvre variable, traduit une réalité propre à quelques sites ou bien à l'ensemble des gisements témoignant de sa capture. Enfin, dans l'hypothèse où la fourrure du Lièvre variable était davantage recherchée, on peut se demander pourquoi si peu de sites témoignent de la capture de cet animal. On ne peut exclure que, dans le cas d'un prélèvement exclusif de la fourrure, les lièvres aient été dépouillés sur le lieu de leur capture et abandonnés sur place. On peut aussi expliquer cette rareté, non pas par un manque de visibilité relatif à une exploitation hors site, mais par un statut particulier de la fourrure de Lièvre, peut-être un produit rare et réservé comme il en existe dans toutes les populations de chasseurscueilleurs actuels. Enfin, rappelons que certains problèmes d'ordre méthodologique rendent difficile l'interprétation des données, si précises soient-elles. Les nouvelles études entreprises, notamment celle des restes de Lièvre variable du Kesslerloch (Napierala, en cours), apporteront probablement des éclaircissements de cet ordre.

\section{Remerciements}

Toute notre gratitude va à celles et ceux qui ont contribué à la réalisation de cette étude ou de cet article : Christine Boussat, Anne Bridault, Jacques-Élie Brochier, JeanChristophe Castel, Christophe Delage, Philippe Jugie, Véronique Laroulandie, Stéphane Madelaine, Hannes Napierala, Bemard Nicolas, Raymond Panattoni, Manuel Perez Ripoll, François Poplin, Stéphane Renault

\section{BIBLIOGRAPHIE}

ALBRECHT G., BERKE H., POPLIN F. 1983 - Restes de mammifères du Petersfels $P 1$ et Petersfels $P 3$, fouilles 1974-1976. In : G. Albrecht, H. Berke, F. Poplin (Ed.), Recherches scientifiques sur les inventaires magdaléniens du Petersfels, fouilles 1974-1976. Verlag Archaeologica Venatoria, Institut für Urgeschichte der Universität Tübingen, p. 63-127

AURA TORTOSA J.E., VILLAVERDE BONILLA V., PEREZ RIPOLL M., MARTINEZ VALLE R., GUILLEM CALATAYUD P. 2002 - Big Game and Small Prey: Paleolithic and Epipaleolithic Economy From Valenci (Spain). Joumal of Archaeological Method and Theory, 9, 3, p. 215-268

AVERBOUH A. et CLEYET-MERLE J.-J. 1995 - 3. Hameçons. In : A. Averbouh, C. Bellier, A. Billamboz, P. Cattelain, J.-J. Cleyet-Merle, M. Julien, L. Mons, D. Ramseyer, M.-R. Séronie-Vivien, A.-C. Welté (Ed.), Eléments barbelés et apparentés, Fiches typologiques de l'industrie de l'os préhistorique, cahier VII, Treignes : CEDARC, p. 83-99

AVERBOUH A. 2003 - Les tubes. In : J. Clottes \& H. Delporte (Ed.), La grotte de la Vache (Ariège), 1. Les occupations du Magdalénien. Documents préhistoriques, 16, Paris : co-éd. du CTHS/RMN, p. 343-352.

BARONE R., PAVAUX C., BLIN P.-C., COQ P. 1973 - Atlas d'anatomie du Lapin. Paris : Masson \& Cie, 64 p., ill.

BELLIER C., CATTELAIN P., WELTÉ A.-C. 1995 - 2. Foënes. In : A. Averbouh, C. Bellier, A. Billamboz, P. Cattelain, J.-J. Cleyet-Merle, M. Julien, L. Mons, D. Ramseyer, M.-R. Séronie-Vivien, A.-C. Welté (Ed.), Eléments barbelés et apparentés, Fiches typologiques de l'industrie de l'os préhistorique, cahier VII, Treignes : CEDARC, p. 67-82

BONNISSENT D. 1993 - Choix et exploitation des bois de renne sur le site de La Madeleine (Dordogne). Bordeaux : Université de Bordeaux I, 1993. 1 t., 48 p. ill. 
BOUCHUD J. 1966 - Essai sur le Renne et la climatologie du Paléolithique moyen et supérieur. Périgueux : Imprimerie R. et M. Magne, 300 p., ill.

BOUVIER J.-M. 1972 - Les restes humains de La Madeleine (Dordogne). Bulletin de la Société d'Anthropologie du Sud-Ouest, VIII, p. 1-10

BOUVIER J.-M. 1973 - Nouvelle diagnose stratigraphique du gisement éponyme de La Madeleine (Tursac, Dordogne). CR de l'Académie des Sciences de Paris, CCLXXVII, p. 2625-2628

BOUVIER J.-M. 1974 - Sagaies du Magdalénien supérieur de plusieurs gisements de Charente et Périgord. In : $\mathrm{H}$. Camps-Fabrer (Ed.), Premier colloque international sur l'industrie de l'os préhistorique. Actes du colloque de l'Abbaye de Sénanque (1974), Aix-en-Provence : Editions de l'Université de Provence, p. 181-185.

BOUVIER J.-M. 1977 - Un gisement préhistorique : La Madeleine. Périgueux : Fanlac, 86 p., ill.

BOUVIER J.-M. 1979 - La Madeleine : acquis récents. In : D. De Soneville-Bordes (Ed.), La fin des temps glaciaires en Europe. Chronostratigraphie et écologie des cultures du Paléolithique final. Actes du colloque de Talence (1977), Paris : CNRS, p. 435-441

BREUIL H. 1937 - Les subdivisions du Paléolithique supérieur et leur signification. Congrès intemational d'anthropologie et d'archéologie préhistoriques. Compte-rendu de la XIVè session, Genève 1912, 2ème édition, Genève, p. 5-78

BRIDAULT A. 1996 - Le problème de l'exploitation du gibier au Tureau des Gardes (Marolles-sur-Seine, Seineet-Mame). In : Y. Pautrat (Ed.), Paléolithique supérieur et Epipaléolithique dans le Nord-Est de la France. Actes de la table ronde de Dijon (1995), Dijon : Cahiers Archéologiques de Bourgogne, 6, p. 141-151

BULLINGER J., LEESCH D., PLUMETTAZ N. 2006 - Le site magdalénien de Monruz, 1. Premiers éléments pour l'analyse d'un habitat de plein air. Neuchâtel : Archéologie neuchâteloise, 33, p. 227 p., ill.

BUNDGEN B. 2002 - Évolution des comportements techniques au Magdalénien supérieur : les données de l'industrie lithique de La Madeleine (Dordogne), séries récentes. Bordeaux : Université de Bordeaux I, 2002. 1 t., 245 p., ill.

CAPITAN L. et PEYRONY D. 1928 - La Madeleine, son gisement, son industrie, ses oeuvres d'art. Paris : Publications de I'Institut International d'Anthropologie, 2, Librairie Nourry, 125 p., ill.

CASTEL J.-C. 1999 - Comportements de subsistance au Solutréen et au Badegoulien d'après les faunes de Combe Saunière (Dordogne) et du Cuzoul de Vers (Lot). Bordeaux : Université de Bordeaux I, 1999. 2 t., 619 p., ill.
CASTEL J.-C., LIOLIOS D., CHADELLE J.-P., GENESTE J.-M. 1998 - De l'alimentaire et du technique : la consommation du renne dans le Solutréen de la grotte de CombeSaunière. In : J.- Ph. Brugal, L. Meignen \& M. Patou-Mathis (Ed.), Economie préhistorique : les comportements de subsistance au paléolithique. Actes des XVIIlè rencontres internationales d'archéologie et d'histoire d'Antibes (1997), Antibes : APDCA, p. 433-450

CATTELAIN P. 1988 - P ropulseurs. Fiches typologiques de l'industrie osseuse préhistorique, cahier II, Aix-enProvence : Publications de l'Université de Provence.

CHARLES R. \& JACOBI R.M. 1994 - The lateglacial fauna from the Robin Hood cave, Cresswell crags: a re-assessment. Oxford Journal of Archaeology, 13, 1, p. 1-32

CHAUVIÈRE F.-X. et CASTEL J.-C. 2004 - Le statut du renard à Combe-Saunière (Dordogne, France) et dans le Solutréen de l'Est aquitain. In : J.-Ph. Brugal \& J. Desse (Ed.), Petits animaux et sociétés humaines. Du complément alimentaire aux ressources utilitaires. Actes des XXIVè rencontres internationales d'archéologie et d'histoire d'Antibes, Antibes (2003), APDCA, p. 389-402

CHAUVIÈRE F.-X. et FONTANA L. 2005 - L'exploitation des rennes du Blot (Haute-Loire) : entre subsistance, technique et symbolique. In : V. Dujardin (Ed.), Industrie osseuse et panure du Solutréen au Magdalénien en Europe. Actes de la Table ronde sur le Paléolithique supérieur récent, Angoulême (2003), Mémoire de la Société Préhistorique Française XXXIX, p. 137-147

CHAUVIÈRE F.-X. et FONTANA L. sous presse Exploitation du monde animal du Châtelperronien au Mésolithique dans le Massif Central : études anciennes, approches récentes. In : J.-P. Raynal (Ed), Un siècle de Préhistoire et de Protohistoire dans le Massif Central. Actes du Colloque SPF, Le Puy-en-Velay (2004)

CHAUVIÈRE F.-X., FONTANA L., LANG, avec la collaboration de BONANI, G. et HAJDAS I. 2006 - Une préhampe magdalénienne en bois de renne aux Petits Guinards (Allier, France). CR de l'Académie des Sciences, section Palévol., 5, p. 725-733

COCHARD D. 2004 - Les léporidés dans la subsistance paléolithique du sud de la France. Bordeaux : Université de Bordeaux I, 2004. 1 t., 356 p., ill.

DELPECH F. 1983 - Les faunes du Paléolithique supérieur dans le sud-ouest de la France. Cahiers du Quatemaire, 6, Paris : CNRS, 453 p., ill.

DELPECH F. et LE GALL O. 1983 - La faune magdalénienne de la grotte des Eglises (Ussat, Ariège). Bulletin de la Société Préhistorique de l'Ariège, 38, p. 91-118

DELPORTE H., HAHN J., MONS L., PINÇON G., DE SONNEVILLE-BORDES D. 1988 - Sagaies. Fiches typologiques 
de l'industrie osseuse préhistorique cahier 1, Aix-enProvence : Publications de l'Université de Provence.

DONARD E. 1982 - Recherche sur les léporinés quaternaires (Pléistocène moyen et supérieur, Holocène). Bordeaux : Université de Bordeaux I, 1982. 2 t., 161 p., ill.

FONTANA L. 1998a - Mobilité et subsistance au Magdalénien dans le Languedoc occidental et le Roussillon. Paris : Université de Paris I, 1998. 2 t., 287 p., ill.

FONTANA L. 1998b - Subsistance et territoire au Magdalénien supérieur dans les Pyrénées : l'apport des données archéozoologiques de la grotte de Belvis (Aude). Préhistoiredu Sud-Ouest, 5, 2, p. 131-146

FONTANA L. 1999 - Mobilité et subsistance au Magdalénien dans le Bassin de l'Aude. Bulletin de la Société Préhistorique Française, 96, 2, p.175-190

FONTANA L. 2000 - La chasse au Renne au Paléolithique supérieur : nouvelles voies de recherche. Paléo, 12, p. 141-164

FONTANA L. 2001 - Étude archéozoologique des collections du Fourneau du Diable (Bourdeilles, Dordogne) : un exemple du potentiel des faunes paléolithiques issues de fouilles anciennes. Paléo, 13, p. 159-182

FONTANA L. 2003 - Characterization and exploitation of the Arctic Hare (Lepus timidus) during the Magdalenian: surprising data from Gazel Cave (Aude, France)? In : S. Costamagno \& V. Laroulandie (Ed.), Mode de vie au Magdalénien : Apports de l'archéozoologie. Actes du XIVè Congrès UISPP de Liège (2001), BAR Int. Ser. 1144 (Archaeopress), p. 101-118

FONTANA L. 2004 - Le statut du Lièvre variable (Lepus timidus) en Europe occidentale au Magdalénien : premier bilan et perspectives. In : J.-Ph. Brugal et J. Desse (Ed.), Petits animaux et sociétés humaines. Du complément alimentaire aux ressources utilitaires. Actes des XXIVè rencontres internationales d'archéologie et d'histoire d'Antibes (2003), Antibes : APDCA, p. 297-312

FONTANA L. \& CHAUVIÈRE F.-X. 2007 - Modes d'acquisition et d'exploitation des ressources. In : S.-A. de Beaune (Ed.), Chasseurs-cueilleurs. Comment vivaient nos ancêtres du Paléolithique supérieur. Paris : CNRS Editions, p. 117-130

FRADET C. 2005 - Le travail de l'ivoire au Paléolithique supérieur: l'exemple du Magdalénien. Toulouse : Université de Toulouse II Le Mirail, 2005. 90 p., ill.

HAWS J.A. 2003 - An investigation of the Late Upper Palaeolithic and Epipalaeolithic hunter-gathere subsistence and settlement patterns in Central Portugal. University of Wisconsin-Madison, 2003, 359 p., ill.

HESCHELER K. 1907 - Die Tierreste im Kesslerloch bei Thayngen. Neue Denkschriften Schweiz, 43, p. 61-154
HEIERLI J. 1907 - Des Kesslerloch bei Thaingen. Zürich : Zürcher \& Furrer, 213 p.,ill

HOCKETT B.S. 1991 - Toward distinguish human and raptor patterning on leporid bones. American Antiquity, 56 , p. $667-679$

HOCKETT B.S. 1992 - Faunal Remains. In: A. Du Barton (Ed.), A Gabled Wooden Lodge in An Archaeological Context: Archaeological Investigations at Sample Unit U19adPL, Nevada Test Site, Nye County, Nevada. Las Vegas: Desert Research Institute, Quatemary Sciences Center, p. 67-75

HOCKETT B.S. 1994 - A Descriptive Reanalysis of the Leporid Bones from Hogup Cave, Utah. Joumal of Califomia and Great Basin Anthropology, 16, p. 106-117

HOCKETT B.S. 1995 - Comparison of leporid bones in raptor pellets, raptor nests, and archaeological sites in the Great Basin. North American Archaeologist, 16, p. 223-238

HOCKETT B.S. \& BICHO N.F. 2000 - The Rabbits of Picareiro Cave: Small Mammal Hunting During the Late Upper Palaeolithic in the Portuguese Estremadura. Journal of Archaeological Science, 27, p. 715-723

HOCKETT B.S. \& J.A. HAWS 2002 - Taphonomic and methodological perspectives of leporid hunting during the Upper Palaeolithic of the Western Mediterranean Basin. Journal of Archaeological Method and Theory, 9, p. 269-302

HÖNEISEN M. \& PEYER S. 1994 - Schweizersbild - ein Jägerlager der Späteiszeit Beiträge und Dokumente zur Ausgrabung vor 100 Jahren. Schaffhausen : Schaffhauser Archäologie 2, 236 p., ill.

JOHNSON E. 1985 - Current developments in bone technology. In: M.B. Schiffer (Ed.), Advances in Archaeological Method and Theory, 8, New York: Academic Press, p. 157-235

JULIEN M. 1982 - Les harpons magdaléniens. Supplément à Gallia Préhistoire XVII, Paris : CNRS, 288 p., ill.

JULIEN M. 1995 - Harpons magdaléniens. In : A. Averbouh, C. Bellier, A. Billamboz, P. Cattelain, J.-J. Cleyet-Merle, M. Julien, L. Mons, D. Ramseyer, M.-R. Séronie-Vivien, A.-C. Welté (Ed.), Eléments barbelés et apparentés, Fiches typologiques de l'industrie de l'os préhistorique, cahier VII, Treignes : CEDARC, p. 13-28

LARTET E. et CHRISTY H. 1875 - Reliquiae Aquitanicae. Being contributions to the Archaeology and Palaeontology of Périgord and adjoining provinces of Southern France (1864-1875). Londres : Rupert Jones, ill.

LAVILLE H. 1975 - Climatologie et chronologie du Paléolithique supérieur en Périgord. Études quatemaires, 4, Marseille : Université de Provence, 422 p., ill. 
LEESCH D. 1997 - Un campement magdalénien au bord du lac de Neuchâtel. Cadre chronologique et culturel, mobilier et structure, analyse spatiale (secteur 1). Neuchâtel : Archéologie neuchâteloise, 19, 270 p., ill.

MADELAINE S. 1989 - Contribution des anciennes fouilles à la connaissance des ongulés et de leur milieu durant le Würm récent en Dordogne. Paléo, 1, p. 36-46

MOREL P. et MÜLLER W. 1997 - Un campement magdalénien au bord du lac de Neuchâtel. Étude archéozoologique. Neuchâtel : Archéologie neuchâteloise, 23, 149 p., ill. MORLAN R.E. 1984 - Toward the definition of criteria for the recognition of artificial bone alteration. Quatemary Research, 22, p. 160-171

MÜLLER W. 2004 - One Horse or a Hundred Hares? Small game exploitation in an Upper Palaeolithic context. In : J.-Ph. Brugal \& J. Desse (Ed.), Petits animaux et sociétés humaines. Du complément alimentaire aux ressources utilitaires. Actes des XXIVè rencontres internationales d'archéologie et d'histoire d'Antibes (2003), Antibes : APDCA, p. 489-498

NÜECH J. 1896 - Das Schweizersbild, eine Niderlassung aus palaeolithischer und neolithischer Zeit. Zürich : Zürcher \& Furrer, 337 p., ill.

PELTIER A. 1992 - Fiche générale des bâtons percés. In : H. Barge-Mahieu, H. Camps-Fabrer, V. Feruglio, A. Peltier, D. Ramseyer (Ed.), Bâtons percés, baguettes, Fiches typologiques de l'industrie osseuse préhistorique, Cahier V, Treignes : CEDARC, p. 7-34.

PEREZ RIPOLL M. 1993 - Las marcas tafonomicas en huesos de lagoformos. In : M.P. Fumanal \& J. Bemabeu (Ed.), Estudios sobre Cuatemario. Valencia, p. 227-231

PEREZ RIPOLL M. 2002 - The importance of taphonomic studies of rabbit bones from archaeological sites. In: M. de Renzi et al. (Ed.), Current Topics on Taphonomy and Fossilization. Ayuntamiento de Valencia, p. 499-508

PEREZ RIPOLL M. 2004 - La consommation humaine des lapins pendant le Paléolithique dans la région de Valencia (Espagne) et l'étude des niveaux gravettiens de La Cova de les Cendres (Alicante). In : J.-Ph. Brugal \& J. Desse (Ed.), Petits animaux et sociétés humaines. Du complément alimentaire aux ressources utilitaires. Actes des XXIVè rencontres internationales d'archéologie et d'histoire d'Antibes (2003), Antibes : APDCA, p. 191-206.
PEREZ RIPOLL M. 2005/2006 - Caracterizacion de las fracturas antropicas y sus tipologias en huesos de conejo procedentes de los niveles gravetienses de la Cova de les Cendres (Alicante). Munibe, 57, Homenaje a Jesus Altuna, Tomo I : Paleontologia y Arqueozoologia, p. 239-254

PEREZ RIPOLL M. 2006 - Estudio tafonomico de los huesos de conejo de Falguera. In : O. Garcia Puchol \& J.E. Aura Tortosa (Ed.), El abric de la Falguiera (Alcoi, Alacant). 8.000 anos de ocupacion humana en la cabecera del rio de Alcoi. Ediciones del Museu d'Alcoi, p. 252-255

PROVENZANO N. 1998 - Fiche générale des objets à biseau distal. In : H. Camps-Fabrer, P. Cattelain, S.-Y. Choï, E. David, J.-L. Pascual-Benito, N. Provenzano, D. Ramseyer (Ed.), Biseaux et tranchants. Fiches typologiques de l'industrie osseuse préhistorique, cahier VIII, Treignes : CEDARC, p. 5-16

SACCHI D. 2003 - Le Magdalénien. Apogée de l'art quatemaire. Paris : La Maison des Roches, collection Histoire de la France préhistorique, 126 p., ill.

SCHMITT D.N. 1990 - Bone artifacts and human remains. In: R.G. Elston \& E.E. Budy (Ed.), The Archaeology of James Creek Shelter. University of Utah: Anthropological Papers, 115, p. 117-127

SPETH J.D. 2000 - Boiling vs Baking and roasting: a taphonomic approach to the recognition of cooking techniques in small animals. In: Rowley-Conwy (Ed.), Animal bones, human societies. Oxford: CBA Research Report, p. 89-105

STORDEUR-YEDID D. 1979 - Les aiguilles à chas au Paléolithique.XIIlè Supplément à Gallia Préhistoire, Paris : CNRS, 215 p., ill.

TAGLIACOZZO A. \& FIORE I. 1998 - Butchering of small mammals in the Epigravettian levels of the Romanelli Cave (Apulia, Italy). In : J.- Ph. Brugal, L. Meignen \& M. PatouMathis (Ed.), Economie préhistorique : les comportements de subsistance au Paléolithique. Actes des XVIIlè rencontres internationales d'archéologie et d'histoire d'Antibes (1997), Sophia-Antipolis : APDCA, p.413-424.

TABORIN Y. 1993 - La panure en coquillage au Paléolithique. XXIXè Supplément à Gallia Préhistoire, Paris : CNRS, 538 p. ill. 